\title{
On the first eigenpair of singularly perturbed operators with oscillating coefficients
}

\author{
A. Piatnitski \\ Narvik University College, Postboks 385, 8505 Narvik, Norway, \\ P. N. Lebedev Physical Institute of RAS, 53, Leninski pr., Moscow 119991, Russia, \\ e-mail: andrey@sci.lebedev.ru \\ V. Rybalko \\ Mathematical Division, B.Verkin Institute for Low Temperature Physics \\ and Engineering of the NASU, 47 Lenin ave., Kharkov 61103, Ukraine, \\ e-mail: vrybalko@ilt.kharkov.ua
}

December 20, 2018

The paper deals with a Dirichlet spectral problem for a singularly perturbed second order elliptic operator with rapidly oscillating locally periodic coefficients. We study the limit behaviour of the first eigenpair (ground state) of this problem. The main tool in deriving the limit (effective) problem is the viscosity solutions technique for HamiltonJacobi equations. The effective problem need not have a unique solution. We study the non-uniqueness issue in a particular case of zero potential and construct the higher order term of the ground state asymptotics.

\section{Introduction}

Given a singularly perturbed elliptic operator of the form

$$
\mathcal{L}_{\varepsilon} u=\varepsilon^{2} a^{i j}\left(x, x / \varepsilon^{\alpha}\right) \frac{\partial^{2} u}{\partial x_{i} \partial x_{j}}+\varepsilon b^{j}\left(x, x / \varepsilon^{\alpha}\right) \frac{\partial u}{\partial x_{j}}+c\left(x, x / \varepsilon^{\alpha}\right) u
$$

with a small parameter $\varepsilon>0$, we consider a Dirichlet spectral problem

$$
\mathcal{L}_{\varepsilon} u=\lambda u, \quad u=0 \quad \text { on } \partial \Omega
$$

\footnotetext{
${ }^{0}$ Mathematics Subject Classification 2000:
} 
stated in a smooth bounded domain $\Omega \subset \mathbb{R}^{N}$. We assume that the coefficients $a^{i j}(x, y), b^{j}(x, y)$ and $c(x, y)$ are sufficiently regular functions periodic in $y$ variable, and that $a^{i j}(x, y)$ satisfy the uniform ellipticity condition. Finally, $\alpha>0$ is a fixed positive parameter. Let us remark that in the underlying convection-diffusion model $\varepsilon$ represents characteristic ratio between the diffusion and convection coefficients, while $\varepsilon^{\alpha}$ refers to the microstructure period.

As well known, the operator $\mathcal{L}_{\varepsilon}$ has a discrete spectrum, and the first eigenvalue $\lambda_{\varepsilon}$ (the eigenvalue with the maximal real part) is real and simple; the corresponding eigenfunction $u_{\varepsilon}$ can be chosen to satisfy $u_{\varepsilon}>0$ in $\Omega$. The goal of this work is to study the asymptotic behavior of $\lambda_{\varepsilon}$ and $u_{\varepsilon}$ as $\varepsilon \rightarrow 0$.

The first eigenpair (ground state) of (1.1) plays a crucial role when studying the large time behavior of solutions to the corresponding parabolic initial boundary problem. The first eigenvalue characterizes an exponential growth or decay of a typical solution, as $t \rightarrow \infty$, while the corresponding eigenfunction describes the limit profile of a normalized solution.

Also, since in a typical case the first eigenfunction shows a singular behavior, as $\varepsilon \rightarrow 0$, in many applications it is important to know the set of concentration points of $u_{\varepsilon}$, the so-called hot spots. This concentration set might consist of one point, or finite number of points, or a surface of positive codimension, or it might have more complicated structure. An interesting discussion on hot spots can be found in [38].

Boundary value problems for singularly perturbed elliptic operators have been widely studied in the existing literature. An important contribution to this topic has been done in the classical work [42] that deals with singular perturbed operators with smooth non-oscillating coefficients under the assumption that for $\varepsilon=0$ the problem remains (in a certain sense) well-posed.

The Dirichlet problem for a convection-diffusion operator with a small diffusion and with a convection directed outward at the domain boundary was studied for the first time in [19]. The approach developed in that work relies on large deviation results for trajectories of a diffusion process being a solution of the corresponding stochastic differential equation.

The probabilistic interpretation of solutions and the aforementioned large deviation principle have also been used in [23], 24], [15], where the first eigenvalue is studied for a second order elliptic operator being a singular perturbation of a first order operator.

There are two natural approaches that can be used for studying the logarithmic asymptotics of the principal eigenfunction of a second order singularly perturbed operator. One of them relies on the above mentioned large deviation results for diffusion processes with a small diffusion coefficients. This method was used in [35] for studying operators with smooth coefficients on a compact Riemannian manifolds.

We follow yet another (deterministic) approach based on the viscosity solution techniques for nonlinear PDEs. In the context of linear singularly perturbed equations, these techniques were originally developed in [18] and followed by [5], [21], [34], [9], [10] and other works (see also a review in [4]). Since $u_{\varepsilon}>0$ in $\Omega$, we can represent $u_{\varepsilon}$ as $u_{\varepsilon}(x)=e^{-W_{\varepsilon}(x) / \varepsilon}$ to find that 
$W_{\varepsilon}$ satisfies

$$
-\varepsilon a^{i j}\left(x, x / \varepsilon^{\alpha}\right) \frac{\partial^{2} W_{\varepsilon}}{\partial x_{i} \partial x_{j}}+H\left(\nabla W_{\varepsilon}, x, x / \varepsilon^{\alpha}\right)=\lambda_{\varepsilon}
$$

with $H(p, x, y)=a^{i j}(x, y) p_{i} p_{j}-b^{j}(x, y) p_{j}+c(x, y)$, and the Dirichlet boundary condition for $u_{\varepsilon}$ yields $W_{\varepsilon}=+\infty$ on $\partial \Omega$. Using perturbed test functions we pass to the limit in (1.2) and get the limit Hamilton-Jacobi equation of the form

$$
\bar{H}(\nabla W(x), x)=\lambda \quad \text { in } \Omega .
$$

with an effective Hamiltonian $\bar{H}(p, x)$ whose definition depends on whether $\alpha>1, \alpha=1$ or $0<\alpha<1$. We show that in the limit $\varepsilon \rightarrow 0$ the boundary condition $W_{\varepsilon}=+\infty$ on $\partial \Omega$ yields

$$
\bar{H}(\nabla W(x), x) \geq \lambda \quad \text { on } \partial \Omega
$$

The latter condition is known [40, [12] as the state constraint boundary condition. Both equation (1.3) and boundary condition (1.4) are understood in viscosity sense.

Equations of type (1.2) have been extensively studied in the existing literature. One can find a short review of state of the art in [29], 25] and in more recent works [8], [1], see also references therein.

Earlier, singularly perturbed KPP-type reaction-diffusion equations were studied in [32] where, in particular, equations with rapidly oscillating coefficients were considered. It was shown that the classical Huygens principle might fail to work in this case.

In the present work, deriving the effective problem (1.3)-(1.4) relies on the idea of perturbed test functions originally proposed in [16]. We strongly believe that with the help of the techniques developed recently in [29], [26], [30], [1] this result can be extended to a more general almost periodic setting as well as random stationary ergodic setting. In other words, the periodicity assumption can be replaced with the assumption that the coefficients in (1.1) are almost periodic or random statistically homogeneous and ergodic with respect to the fast variable, at least in the case $\alpha=1$. The case $\alpha \neq 1$ looks more difficult and might require some extra assumptions. We refer to [41, [39], [7, [29], 26], [8], [1] for (far not complete list of) various results on almost periodic and random homogenization of nonlinear PDEs. However, the essential novelty of this work comes in the (logically) second part of the paper devoted to the improved ground state asymptotics and resolving the non-uniqueness issue for (1.3)-(1.4). The generalization of this part to non-periodic settings is an open problem.

Problem (1.3) -(1.4) is known as ergodic or additive eigenvalue problem. Its solvability was first proved in [31] in periodic setting, more recent results are contained, e.g., in [22] as well as in [14], [2] where stationary ergodic Hamiltonians were considered. There exists the unique additive eigenvalue $\lambda$ of (1.3)-(1.4) while the eigenfunction $W$ need not be unique even up to an additive constant. This non-uniqueness issue is intimately related to the structure of the so-called Aubry set of effective Hamiltonian which play the role of a hidden boundary for (1.3) $-(1.4)$. Loosely speaking the non-uniqueness in (1.3)-(1.4) appears when the Aubry set is 
disconnected. By contrast, for every $\varepsilon>0$ the eigenfunction $u_{\varepsilon}$ is unique up to a normalization, and it is natural to find true limit of $W_{\varepsilon}=-\varepsilon \log u_{\varepsilon}$ among solutions of (1.3) $-(1.4)$. This challenging problem is addressed in a particular case of (1.1) with $c(x, y)=0, \alpha \geq 1$. Following [36] we introduce the effective drift (convection) and assume that it has a finite number of hyperbolic fixed points in $\Omega$, and that the Aubry set of the effective Hamiltonian coincides with this finite collection of points. It follows from our results that in this case $\lambda_{\varepsilon}$ tends to zero as $\varepsilon \rightarrow 0$. We show that $\lambda_{\varepsilon} / \varepsilon$ has a finite limit that can be determined in terms of eigenvalues of Ornstein-Uhlenbeck operators in $\mathbb{R}^{N}$ obtained via local analysis of (1.1) at the scale $\sqrt{\varepsilon}$ in the vicinity of aforementioned fixed points. This, in turn, enables fine selection of the additive eigenfunction corresponding to $\lim _{\varepsilon \rightarrow 0} W_{\varepsilon}$.

\section{Main results}

We begin with standing hypotheses which are assumed to hold throughout this paper. We assume that $\Omega$ is connected and has $C^{2}$ boundary $\partial \Omega$; the coefficients $a^{i j}(x, y), b^{j}(x, y), c(x, y) \in$

$C^{1}\left(\bar{\Omega} \times \mathbb{R}^{N}\right)$ are $Y$-periodic in $y$ functions, where $Y=(0,1)^{N}$. The matrix $\left(a^{i j}\right)_{i, j=1, N}$ is uniformly positive definite:

$$
a^{i j}(x, y) \zeta_{i} \zeta_{j} \geq m|\zeta|^{2}>0 \quad \forall \zeta \neq 0,
$$

and, without loss of generality, we can assume the symmetry $a^{i j}=a^{j i}$.

The first eigenfunction $u_{\varepsilon}$ of the operator (1.1) can be normalized to satisfy

$$
1=\max _{\Omega} u_{\varepsilon}\left(u_{\varepsilon}>0 \text { in } \Omega\right),
$$

then its scaled logarithmic transformation

$$
W_{\varepsilon}:=-\varepsilon \log u_{\varepsilon}
$$

is a nonnegative function vanishing at the points of maxima of $u_{\varepsilon}$.

The asymptotic behavior of $\lambda_{\varepsilon}$ and $W_{\varepsilon}$ is described in

Theorem 1. The eigenvalues $\lambda_{\varepsilon}$ converge as $\varepsilon \rightarrow 0$ to the limit $\lambda$, which is the unique real number for which problem (1.3), (1.4) has a continuous viscosity solution. The functions $W_{\varepsilon}$ converge (up to extracting a subsequence) to a limit $W$ uniformly on compacts in $\Omega$, and every limit function $W$ is a viscosity solution of (1.3), (1.4).

The effective Hamiltonian $\bar{H}(p, x)$ in (1.3) is given by the following formulas, depending on the parameter $\alpha$.

(i) If $\alpha>1$ then

$$
\bar{H}(p, x)=\int_{Y} H(p, x, y) \vartheta(y) \mathrm{d} y
$$


where

$$
H(p, x, y)=a^{i j}(x, y) p_{i} p_{j}-b^{j}(x, y) p_{j}+c(x, y),
$$

and $\vartheta(y)$ is the unique $Y$-periodic solution of the equation $\frac{\partial^{2}}{\partial y_{i} \partial y_{j}}\left(a^{i j}(x, y) \vartheta\right)=0$ normalized by $\int_{Y} \vartheta(y) \mathrm{d} y=1$.

(ii) If $\alpha=1$ then $\bar{H}(p, x)$ is the first eigenvalue (eigenvalue with the maximal real part) of the problem

$$
\begin{aligned}
& a^{i j}(x, y) \frac{\partial^{2} \vartheta}{\partial y_{j} \partial y_{j}}+\left(b^{j}(x, y)-2 a^{i j}(x, y) p_{i}\right) \frac{\partial \vartheta}{\partial y_{j}}+H(p, x, y) \vartheta=\bar{H}(p, x) \vartheta, \\
& \vartheta(y) \quad \text { is } Y \text {-periodic. }
\end{aligned}
$$

According to the Krein-Rutman theorem $\bar{H}(p, x)$ is real.

(iii) If $0<\alpha<1$ then $\bar{H}(p, x)$ is the unique number such that the problem

$$
H(p+\nabla \vartheta(y), x, y)=\bar{H}(p, x)
$$

has a $Y$-periodic viscosity solution $\vartheta(y)$; here $p \in \mathbb{R}^{N}$ and $x \in \bar{\Omega}$ are parameters.

We note that the effective Hamiltonian $\bar{H}(p, x)$ is continuous on $\mathbb{R}^{N} \times \bar{\Omega}$, convex in $p$ and coercive, moreover $\bar{H}(p, x) \geq m_{1}|p|^{2}-C, m_{1}>0$. The viscosity solutions theory for such Hamiltonians is well established. Following [22] (see also [33]) we present various representation formulas for the solutions of problem (1.3)-(1.4).

Let us rewrite problem (1.3)-(1.4) in the form

$$
\begin{array}{ll}
\bar{H}(\nabla W(x), x) \leq \lambda & \text { in } \Omega \\
\bar{H}(\nabla W(x), x) \geq \lambda & \text { in } \bar{\Omega},
\end{array}
$$

i.e. (2.6) requires that $W$ is a viscosity subsolution in $\Omega$ while (2.7) means that $W$ is a viscosity supersolution in $\bar{\Omega}$. Then the number $\lambda_{\bar{H}}$ (additive eigenvalue) for which (1.3)-(1.4) has a solution is given by

$$
\lambda_{\bar{H}}=\inf \{\lambda ;(2.6) \text { has a solution } W \in C(\bar{\Omega})\} .
$$

It can also be expressed in terms of action minimization,

$$
\lambda_{\bar{H}}=-\lim _{t \rightarrow \infty} \frac{1}{t} \inf \int_{0}^{t} \bar{L}(\dot{\eta}, \eta) \mathrm{d} \tau,
$$

where the infimum is taken over absolutely continuous curves $\eta:[0, t] \rightarrow \bar{\Omega}$, and $\bar{L}(v, x)$ is the Legendre transform of $\bar{H}(p, x)$,

$$
\bar{L}(v, x)=\max \{v \cdot p-\bar{H}(p, x)\} .
$$

Let us define now the distance function

$$
d_{\bar{H}-\lambda_{\bar{H}}}(x, y)=\sup \left\{W(x)-W(y) ; W \in C(\bar{\Omega}) \text { is a solution of (2.6) for } \lambda=\lambda_{\bar{H}}\right\} .
$$


It is known (see, e.g., [22]) that $d_{\bar{H}-\lambda_{\bar{H}}}(x, x)=0, d_{\bar{H}-\lambda_{\bar{H}}}(x, y)$ is Lipschitz continuous, $d_{\bar{H}-\lambda_{\bar{H}}}(x, y) \leq d_{\bar{H}-\lambda_{\bar{H}}}(x, z)+d_{\bar{H}-\lambda_{\bar{H}}}(z, y)$. Besides, for every $y \in \bar{\Omega}$ the function $d_{\bar{H}-\lambda_{0}}(x, y)$ is a solution of (2.6) for $\lambda=\lambda_{\bar{H}}$ and $\bar{H}\left(\nabla_{x} d_{\bar{H}-\lambda_{\bar{H}}}(x, y), x\right) \geq \lambda_{\bar{H}}$ in $\bar{\Omega} \backslash\{y\}$. The number $\lambda_{\bar{H}}$ is such that the Aubry set $\mathcal{A}_{\bar{H}-\lambda_{\bar{H}}}$,

$$
\mathcal{A}_{\bar{H}-\lambda_{\bar{H}}}=\left\{y \in \bar{\Omega} ; d_{\bar{H}-\lambda_{\bar{H}}}(x, y) \text { is a solution of (2.7) for } \lambda=\lambda_{\bar{H}}\right\} \text {, }
$$

is nonempty. Note also that the distance function $d_{\bar{H}-\lambda_{\bar{H}}}(x, y)$ admits the representation

$$
d_{\bar{H}-\lambda_{\bar{H}}}(x, y)=\inf \left\{\int_{0}^{t}\left(\bar{L}(\dot{\eta}, \eta)+\lambda_{\bar{H}}\right) \mathrm{d} \tau, \eta(0)=y, \eta(t)=x, t>0\right\},
$$

and the Aubry set can be characterized by

$$
y \in \mathcal{A}_{\bar{H}-\lambda_{\bar{H}}} \Longleftrightarrow \sup _{\delta>0} \inf \left\{\int_{0}^{t}\left(\bar{L}(\dot{\eta}, \eta)+\lambda_{\bar{H}}\right) \mathrm{d} \tau, \eta(0)=\eta(t)=y, t>\delta\right\}=0 .
$$

The infimum in (2.11) and (2.12) is taken over absolutely continuous curves $\eta:[0, t] \rightarrow \bar{\Omega}$.

According to the definition of $d_{\bar{H}-\lambda_{\bar{H}}}(x, y)$ every solution $W$ of (1.3)-(1.4) satisfies $W(x)-$ $W(y) \leq d_{\bar{H}-\lambda_{\bar{H}}}(x, y)$; this inequality holds, in particular, for all $x, y \in \mathcal{A}_{\bar{H}-\lambda_{\bar{H}}}$. Conversely, given a function $g(x)$ on $\mathcal{A}_{\bar{H}-\lambda_{\bar{H}}}$ which satisfies the compatibility condition $g(x)-g(y) \leq d_{\bar{H}-\lambda_{\bar{H}}}(x, y)$ $\forall x, y \in \mathcal{A}_{\bar{H}-\lambda_{\bar{H}}}$ then

$$
W(x)=\min \left\{d_{\bar{H}-\lambda_{\bar{H}}}(x, y)+g(y) ; y \in \mathcal{A}_{\bar{H}-\lambda_{\bar{H}}}\right\}
$$

is the unique solution of (1.3) -(1.4) for $\lambda=\lambda_{\bar{H}}$ such that $W(x)=g(x)$ on $\mathcal{A}_{\bar{H}-\lambda_{\bar{H}}}$. In Appendix $\mathrm{A}$ we show the following simple uniqueness criterion for problem (1.3)-(1.4): a solution $W$ (for $\lambda=\lambda_{\bar{H}}$ ) is unique up to an additive constant if and only if $S_{\bar{H}-\lambda_{\bar{H}}}(x, y)=$ $0 \forall x, y \in \mathcal{A}_{\bar{H}-\lambda_{\bar{H}}}$, where $S_{\bar{H}-\lambda_{\bar{H}}}(x, y)$ denotes the symmetrized distance, $S_{\bar{H}-\lambda_{\bar{H}}}(x, y)=$ $d_{\bar{H}-\lambda_{\bar{H}}}(x, y)+d_{\bar{H}-\lambda_{\bar{H}}}(y, x)$.

The interesting issue of non-uniqueness in the limit (homogenized) problem can be resolved by studying next terms in the asymptotic expansion of $\lambda_{\varepsilon}$. This question is rather complicated, and we mainly focus in this work on a particular case when $c(x, y)=0$ and $\alpha=1$, so that operator (1.1) takes the form

$$
\mathcal{L}_{\varepsilon} u=\varepsilon^{2} a^{i j}(x, x / \varepsilon) \frac{\partial^{2} u}{\partial x_{i} \partial x_{j}}+\varepsilon b^{j}(x, x / \varepsilon) \frac{\partial u}{\partial x_{j}} .
$$

Moreover we assume that $\lambda_{\bar{H}}=0$ and that the corresponding Aubry set $\mathcal{A}_{\bar{H}}$ has a special structure. The analogous result for $\alpha>1$ is established in Section 8 ,

For $\alpha \geq 1$ the effective Hamiltonian $\bar{H}(p, x)$ is strictly convex in $p$, i.e. $\left(\frac{\partial^{2}}{\partial p_{i} \partial p_{j}} \bar{H}(p, x)\right)_{i, j=\overline{1, N}}$ is positive definite for all $p \in \mathbb{R}^{N}$ and $x \in \bar{\Omega}$, see [11], or [13] for $\alpha=1$, while for $\alpha>1$ the Hamiltonian $\bar{H}(p, x)$ is a quadratic function in $p$. Note also that if $c(x, y)=0$ then $\bar{H}(0, x)=0$. 
Therefore, the Lagrangian $\bar{L}(v, x)$ is strictly convex and $\bar{L}(v, x)=\max \{p \cdot v-\bar{H}(p, x)\} \geq$ $-\bar{H}(0, x)=0$, in the case $c(x, y)=0, \alpha=1$ we are interested in. Thus we have

$$
\bar{L}(v, x) \geq 0, \quad \text { and } \bar{L}(v, x)=0 \Longleftrightarrow v_{j}=\frac{\partial \bar{H}}{\partial p_{j}}(0, x) .
$$

On the other hand direct calculations show that

$$
-\frac{\partial \bar{H}}{\partial p_{j}}(0, x)=\bar{b}^{j}(x):=\int_{Y} b^{j}(x, y) \theta^{*}(x, y) \mathrm{d} y,
$$

the functions $\bar{b}^{j}(x)$ being components of the so-called effective drift $\bar{b}(x)$ defined by the right hand side of (2.15) via the $Y$-periodic solution $\theta^{*}$ of

$$
\frac{\partial^{2}}{\partial y_{i} \partial y_{j}}\left(a^{i j}(x, y) \theta^{*}\right)-\frac{\partial}{\partial y_{j}}\left(b^{j}(x, y) \theta^{*}\right)=0
$$

normalized by $\int_{Y} \theta^{*} \mathrm{~d} y=1$. (Note that $\theta^{*}>0$ and it is a $C^{2}$ function.) Thus the Lagrangian $\bar{L}(v, x)$ can be represented in the form

$$
\bar{L}(v, x)=\kappa \sum\left(v_{j}+\bar{b}^{j}(x)\right)^{2}+\tilde{L}(v, x), \quad \text { where } 0 \leq \tilde{L}(v, x) \leq \tilde{\kappa} \sum\left(v_{j}+\bar{b}^{j}(x)\right)^{2}, 0<\kappa<\tilde{\kappa} .
$$

This implies, in view of (2.12), that the Aubry set $\mathcal{A}_{\bar{H}}$ of the Hamiltonian $\bar{H}$ coincides with that of the Hamiltonian $\sum p_{j}^{2}-\bar{b}^{j}(x) p_{j}$ whose corresponding Lagrangian is $\frac{1}{4} \sum\left(v_{j}+\bar{b}^{j}(x)\right)^{2}$. In particular, the additive eigenvalue $\lambda_{\bar{H}}$ is zero if and only if there is an orbit $\eta: \mathbb{R} \rightarrow \bar{\Omega}$, $\dot{\eta}=-\bar{b}(\eta)$. We moreover assume that

$$
\begin{aligned}
& \mathcal{A}_{\bar{H}} \neq \emptyset \text { and } \mathcal{A}_{\bar{H}} \subset \Omega, \\
& \mathcal{A}_{\bar{H}} \text { is a finite set of hyperbolic fixed points } \xi \text { of the ODE } \dot{x}=-\bar{b}(x) .
\end{aligned}
$$

Under this assumption we are able to study the leading (of order $\varepsilon$ ) term of the asymptotic expansion of $\lambda_{\varepsilon}$. This in turn allows us to establish a sufficient condition for selecting the unique limit of functions $W_{\varepsilon}$ among solutions of the homogenized problem (1.3), (1.4).

Theorem 2. Let $\alpha=1$ and $c(x, y)=0$. Then, under condition (2.17) we have

$$
\lambda_{\varepsilon}=\varepsilon \bar{\sigma}+\bar{o}(\varepsilon), \quad \text { where } \bar{\sigma}=\max \left\{\sigma(\xi) ; \xi \in \mathcal{A}_{\bar{H}}\right\},
$$

and $\sigma(\xi)$ is the sum of negative real parts of the eigenvalues of the matrix

$$
-B^{i j}(\xi)=-\frac{\partial \bar{b}^{j}}{\partial x_{i}}(\xi)
$$

corresponding to the linearized effective drift at $\xi$ (since every fixed point $\xi$ is assumed to be hyperbolic $-B(\xi)$ has no eigenvalues with zero real part). Moreover, if the maximum in (2.18) is attained at exactly one $\xi=\bar{\xi}$ then 
(i) the scaled logarithmic transformations $W_{\varepsilon}=-\varepsilon \log u_{\varepsilon}$ of eigenfunctions $u_{\varepsilon}$ (normalized by (2.2)) converge uniformly on compacts in $\Omega$ to $W(x)=d_{\bar{H}}(x, \bar{\xi})$, i.e. $W$ is the maximal viscosity solution of $\bar{H}(\nabla W(x), x)=0$ in $\Omega, \bar{H}(\nabla W(x), x) \geq 0$ on $\partial \Omega$, such that $W(\bar{\xi})=0$;

(ii) $u_{\varepsilon}(\bar{\xi}+\sqrt{\varepsilon} z) \rightarrow u(z)$ in $C(K)$ and weakly in $H^{1}(K)$ for every compact $K$, and the limit $u$ is the unique positive eigenfunction of the Ornstain-Uhlenbeck operator,

$$
Q^{i j} \frac{\partial^{2} u}{\partial z_{i} \partial z_{j}}+z_{i} B^{i j} \frac{\partial u}{\partial z_{j}}=\bar{\sigma} u \quad \text { in } \mathbb{R}^{N},
$$

normalized by $u(0)=1$ and satisfying the following condition, $u(z) e^{\mu\left|\Pi_{s}^{*} z\right|^{2}-\nu\left|\Pi_{u}^{*} z\right|^{2}}$ is bounded on $\mathbb{R}^{N}$ for some $\mu>0$ and every $\nu>0$. The coefficients in (2.19) are given by $B^{i j}=B^{i j}(\bar{\xi})$, $Q^{i j}=\frac{1}{2} \frac{\partial^{2} \bar{H}}{\partial p_{i} \partial p_{j}}(0, \bar{\xi}) ; \Pi_{s}$ and $\Pi_{u}$ denote spectral projectors on the invariant subspaces of the matrix $B$ corresponding to the eigenvalues with positive and negative real parts (stable and unstable subspaces of the system $\dot{z}_{i}=-B^{i j} z_{j}$ ).

Remark 3. Condition (2.17) is satisfied, in particular, when the vector field $b(x, y)$ is a $C^{1}$ small perturbation of a gradient field $\nabla P(x)$ with $C^{2}$ potential $P(x)$ having the following properties: the set $\{x \in \bar{\Omega} ; \nabla P(x)=0\}$ is formed by a finite collection of points in $\Omega$ and the Hessian matrix $\left(\frac{\partial^{2}}{\partial x_{i} \partial x_{j}} P(x)\right)_{i, j=\overline{1, N}}$ at every such a point is nonsingular (see Appendix (B)).

Condition (2.17) is satisfied if and only if the vector field $\bar{b}$ possesses the following properties:

- $\bar{b}$ has a finite number of fixed points in $\bar{\Omega}$, say $\xi^{1}, \ldots \xi^{n}$. All of them are hyperbolic, and none of them is situated on $\partial \Omega$.

- $\forall y \in \bar{\Omega}$, either $\sup \left\{t<0: x^{y}(t) \notin \bar{\Omega}\right\}>-\infty$, or $\lim _{t \rightarrow-\infty} x^{y}(t)=\xi^{j}$ for some $j \in\{1, \ldots, n\}$, where $x^{y}$ is a solution of the ODE $\dot{x}^{y}=-\bar{b}\left(x^{y}\right), x^{y}(0)=y$.

- there is no any closed path $\xi^{j_{1}}, \xi^{j_{2}}, \ldots, \xi^{j_{k}}=\xi^{j_{1}}$ with $k \geq 2$ such that for any two consecutive points $\xi^{j_{s}}$ and $\xi^{j_{s+1}}$ there is a solution of the equation $\dot{x}=-\bar{b}(x)$ with $\lim _{t \rightarrow-\infty} x(t)=\xi^{j_{s}}$ and $\lim _{t \rightarrow+\infty} x(t)=\xi^{j_{s+1}}$. Note that $\xi^{j_{1}}$ might coincide with $\xi^{j_{2}}$.

Remark 4. It is not hard to show that under condition (2.17) we have $S_{\bar{H}}\left(\xi, \xi^{\prime}\right)>0 \forall \xi, \xi^{\prime} \in$ $\mathcal{A}_{\bar{H}}, \xi \neq \xi^{\prime}$. This means that problem (1.3), (1.4) does have many solutions unless $\mathcal{A}_{\bar{H}}$ is a single point.

Note that condition (2.17) of Theorem 2 assumes, in particular, that all $\omega($ and $\alpha)$-limit points of the ODE $\dot{x}=-\bar{b}(x)$ are fixed points. Another important case, when the ODE $\dot{x}=-\bar{b}(x)$ has limit cycles in $\Omega$ (which is also the case of general position), will be considered in a separate paper.

\section{Singularly perturbed operators in the periodic setting}

Consider the spectral problem for singularly perturbed elliptic operators of the form

$$
\mathcal{L}_{\varepsilon}^{(p e r)} u=\varepsilon^{2} a^{i j}(x) \frac{\partial^{2} u}{\partial x_{i} \partial x_{j}}+\varepsilon b^{j}(x) \frac{\partial u}{\partial x_{j}}+c(x) u,
$$


with $Y$-periodic coefficients $a^{i j}, b^{j}, c \in C^{1}\left(\mathbb{R}^{N}\right), u$ also being $Y$-periodic. We assume the uniform ellipticity condition $a^{i j}(x) \zeta_{i} \zeta_{j} \geq m|\zeta|^{2}>0 \forall \zeta \in \mathbb{R}^{N} \backslash\{0\}$ and the symmetry $a^{i j}=a^{j i}$. Similarly to the case of the Dirichlet boundary condition, the first eigenvalue $\mu_{\varepsilon}$ of $\mathcal{L}_{\varepsilon}^{(p e r)}$ (eigenvalue with the maximal real part) is a real and simple eigenvalue, the corresponding eigenfunction $u_{\varepsilon}$ can be chosen to satisfy $0<u_{\varepsilon}(x) \leq \max u_{\varepsilon}=1$. The asymptotic behavior of $\mu_{\varepsilon}$ as $\varepsilon \rightarrow 0$ and $u_{\varepsilon}$ was studied in [35] using a combination of large deviation and variational techniques. We recover hereafter the results of [35] by means of vanishing viscosity approach and establish as a bi-product some bounds for derivatives of functions $W_{\varepsilon}(x)=-\varepsilon \log u_{\varepsilon}(x)$ that are essential in the proof of Theorem 1 .

First we derive the a priori bounds for the eigenvalues.

Lemma 5. For every $\varepsilon>0$ the eigenvalue $\mu_{\varepsilon}$ of $L_{\varepsilon}^{(\text {per })}$ satisfies the inequalities

$$
\min c(x) \leq \mu_{\varepsilon} \leq \max c(x)
$$

Proof. Let $x^{\prime}$ be a maximum point of $u_{\varepsilon}$, we have

$$
\nabla u_{\varepsilon}\left(x^{\prime}\right)=0, \quad \varepsilon^{2} a^{i j}\left(x^{\prime}\right) \frac{\partial^{2} u_{\varepsilon}}{\partial x_{i} \partial x_{j}}\left(x^{\prime}\right) \leq 0,
$$

therefore $c\left(x^{\prime}\right) u_{\varepsilon}\left(x^{\prime}\right) \geq \mu_{\varepsilon} u_{\varepsilon}\left(x^{\prime}\right)$, i.e. $\mu_{\varepsilon} \leq \max c(x)$. Similarly, if $x^{\prime \prime}$ is a minimum point of $u_{\varepsilon}$ then $\mu_{\varepsilon} u_{\varepsilon}\left(x^{\prime \prime}\right) \geq c\left(x^{\prime \prime}\right) u_{\varepsilon}\left(x^{\prime \prime}\right)$ and therefore $\mu_{\varepsilon} \geq \min c(x)$.

Since $u_{\varepsilon}=e^{-W_{\varepsilon}(x) / \varepsilon}$ we have

$$
-\varepsilon a^{i j}(x) \frac{\partial^{2} W_{\varepsilon}}{\partial x_{i} \partial x_{j}}+a^{i j}(x) \frac{\partial W_{\varepsilon}}{\partial x_{i}} \frac{\partial W_{\varepsilon}}{\partial x_{j}}-b^{j}(x) \frac{\partial W_{\varepsilon}}{\partial x_{j}}+c(x)=\mu_{\varepsilon} .
$$

The bounds for the first and second derivatives of $W_{\varepsilon}(x)$ are obtained in the following

Lemma 6. There is a constant $C$, independent of $\varepsilon$, such that

$$
\max \left|\nabla W_{\varepsilon}\right| \leq C, \quad \max \left|\partial^{2} W_{\varepsilon} / \partial x_{i} \partial x_{j}\right| \leq C / \varepsilon
$$

Proof. The proof of the first bound in (3.4) is borrowed from [18]. Let $D_{1}(x):=\left|\nabla W_{\varepsilon}(x)\right|^{2}$ and $D_{2}(x):=\sum\left|\partial^{2} W_{\varepsilon}(x) / \partial x_{i} \partial x_{j}\right|^{2}$. From (3.3) in conjunction with (3.2) we get $m D_{1} \leq$ $C\left(\varepsilon D_{2}^{1 / 2}+D_{1}^{1 / 2}+1\right)$, this in turn implies that

$$
D_{1} \leq C\left(\varepsilon D_{2}^{1 / 2}+1\right)
$$

Assume that $D_{1}$ attains its maximum at a point $x^{\prime}$, then we have $\nabla D_{1}\left(x^{\prime}\right)=0$ and $a^{i j}\left(x^{\prime}\right) \frac{\partial^{2} D_{1}}{\partial x_{i} \partial x_{j}}\left(x^{\prime}\right) \leq 0$ or

$$
\frac{\partial^{2} W_{\varepsilon}}{\partial x_{i} \partial x_{k}}\left(x^{\prime}\right) \frac{\partial W_{\varepsilon}}{\partial x_{k}}\left(x^{\prime}\right)=0
$$

and

$$
\varepsilon \sum_{k} a^{i j} \frac{\partial^{2} W_{\varepsilon}}{\partial x_{i} \partial x_{k}} \frac{\partial^{2} W_{\varepsilon}}{\partial x_{j} \partial x_{k}} \leq-\varepsilon \sum_{k} a^{i j} \frac{\partial^{3} W_{\varepsilon}}{\partial x_{i} \partial x_{j} \partial x_{k}} \frac{\partial W_{\varepsilon}}{\partial x_{k}} \quad \text { at } x^{\prime}
$$


In order to bound the right hand side of (3.7) we take derivatives of (3.3), this yields

$$
-\varepsilon a^{i j} \frac{\partial^{3} W_{\varepsilon}}{\partial x_{i} \partial x_{j} \partial x_{k}}=\varepsilon \frac{\partial a^{i j}}{\partial x_{k}} \frac{\partial^{2} W_{\varepsilon}}{\partial x_{i} \partial x_{j}}-2 a^{i j} \frac{\partial^{2} W_{\varepsilon}}{\partial x_{i} \partial x_{k}} \frac{\partial W_{\varepsilon}}{\partial x_{j}}+b^{i} \frac{\partial^{2} W_{\varepsilon}}{\partial x_{i} \partial x_{k}}+\frac{\partial b^{i}}{\partial x_{k}} \frac{\partial W_{\varepsilon}}{\partial x_{i}}-\frac{\partial c}{\partial x_{k}} .
$$

Then we multiply (3.8) by $\partial W_{\varepsilon} / \partial x_{k}$, sum up the equations in $k$ and insert the result into (3.7) to obtain

$$
\varepsilon m D_{2}\left(x^{\prime}\right) \leq \varepsilon \sum_{k} a^{i j}\left(x^{\prime}\right) \frac{\partial^{2} W_{\varepsilon}}{\partial x_{i} \partial x_{k}}\left(x^{\prime}\right) \frac{\partial^{2} W_{\varepsilon}}{\partial x_{j} \partial x_{k}}\left(x^{\prime}\right) \leq C\left(\varepsilon D_{1}^{1 / 2}\left(x^{\prime}\right) D_{2}^{1 / 2}\left(x^{\prime}\right)+D_{1}\left(x^{\prime}\right)+D_{1}^{1 / 2}\left(x^{\prime}\right)\right) .
$$

Next we use (3.5) to get that $D_{2}\left(x^{\prime}\right) \leq C / \varepsilon$, and exploiting once more (3.5) we obtain the first bound in (3.4).

To show the second bound in (3.4) we use the following interpolation inequality

$$
\|\nabla u\|_{L^{\infty}}^{2} \leq C\left(\left\|a^{i j} \partial^{2} u / \partial x_{i} \partial x_{j}\right\|_{L^{\infty}}+\|u\|_{L^{\infty}}\right)\|u\|_{L^{\infty}}
$$

which holds for every $Y$-periodic $u$ with a constant $C$ independent of $u$. The proof of this inequality follows the lines of one in the Appendix of [6] (here it is important that the coefficients $a^{i j}$ are Lipschitz continuous). We apply (3.9) to (3.8) to obtain

$$
\left\|\partial^{2} W_{\varepsilon} / \partial x_{l} \partial x_{k}\right\|_{L^{\infty}}^{2} \leq \frac{C}{\varepsilon}\left(\sum\left\|\partial^{2} W_{\varepsilon} / \partial x_{i} \partial x_{j}\right\|_{L^{\infty}}+1\right) \quad \forall l, k,
$$

here we have also used the first bound in (3.4). From (3.10) one easily derives the second bound in (3.4).

It follows from Lemma 5 that $\mu_{\varepsilon} \rightarrow \mu$, up to extracting a subsequence. Due to Lemma 6 the family of functions $W_{\varepsilon}(x)$ is equicontinuous, moreover $\min W_{\varepsilon}(x)=0$ therefore passing to a further subsequence (if necessary) we have $W_{\varepsilon}(x) \rightarrow W(x)$ uniformly. The standard arguments show that the pair $\mu$ and $W$ satisfies the equation

$$
a^{i j}(x) \frac{\partial W}{\partial x_{i}} \frac{\partial W}{\partial x_{j}}-b^{j}(x) \frac{\partial W}{\partial x_{j}}+c(x)=\mu
$$

in the viscosity sense. The number $\mu$ for which (3.11) has a periodic viscosity solution is unique (see [31, [17]), therefore the entire sequence $\mu_{\varepsilon}$ converges to $\mu$ as $\varepsilon \rightarrow 0$.

\section{A priori bounds}

In this section we show that the eigenvalues $\lambda_{\varepsilon}$ of (1.1) are uniformly bounded and the functions $W_{\varepsilon}$ (given by (2) ) uniformly converge on compacts in $\Omega$ as $\varepsilon \rightarrow 0$, up to extracting a subsequence. We also prove an auxiliary result on the behavior of the minimum points of $W_{\varepsilon}-\phi$ (where $\phi$ is an arbitrary $C^{2}$ function) which is important in the subsequent analysis.

Because of the Dirichlet boundary condition on the boundary $\partial \Omega$ and fast oscillations of the coefficients the arguments here are more involved than those in the periodic case. 
Lemma 7. There is a constant $\Lambda$ independent of $\varepsilon$ and such that

$$
-\Lambda \leq \lambda_{\varepsilon} \leq \sup c(x, y) .
$$

Proof. The proof of the upper bound follows by the maximum principle as in Lemma 5 ,

To derive a lower bound for $\lambda_{\varepsilon}$ we construct a function $v_{\varepsilon}$ and choose a number $\Lambda>0$ such that $v_{\varepsilon}=0$ on $\partial \Omega$, and

$$
\mathcal{L}_{\varepsilon} v_{\varepsilon}-\lambda v_{\varepsilon}>0 \quad \text { in } \Omega
$$

for every $\lambda<-\Lambda, 0<\varepsilon<1$. There is a function $W \in C^{2}(\bar{\Omega})$ satisfying the following conditions, $W>0$ in $\Omega$ and $W=0$ on $\partial \Omega,|\nabla W|>1$ in a neighborhood of $\partial \Omega$. Set $v_{\varepsilon}(x):=e^{\kappa W(x) / \varepsilon}-1$, where $\kappa$ is a positive parameter to be chosen later. We assume that $-\Lambda \leq \min c(x, y)$ so that $\lambda<\min c(x, y)$. Then we have

$$
\mathcal{L}_{\varepsilon} v_{\varepsilon}-\lambda v_{\varepsilon} \geq\left(m \kappa^{2}-\kappa\left(M_{1}+\varepsilon M_{2}\right)+\left(c\left(x, x / \varepsilon^{\alpha}\right)-\lambda\right)\right) e^{\kappa W(x) / \varepsilon}-\left(c\left(x, x / \varepsilon^{\alpha}\right)-\lambda\right)>0 \text { in } \Omega^{\prime}
$$

when $\kappa>\kappa_{1}:=\left(M_{1}+M_{2}\right) / m$. Here $\Omega^{\prime}=\{x \in \Omega ;|\nabla W| \geq 1\}, M_{1}=\max \left|b^{i}(x, y) \frac{\partial W}{\partial x_{i}}(x)\right|$, $M_{2}=\max \left|a^{i j}(x, y) \frac{\partial^{2} W}{\partial x_{i} \partial x_{j}}(x)\right|$. On the other hand $\delta:=\inf \left\{W(x) ; x \in \Omega \backslash \Omega^{\prime}\right\}>0$ therefore $e^{\kappa W(x) / \varepsilon}>2$ in $\Omega \backslash \Omega^{\prime}$, when $\kappa>\kappa_{2}:=(\log 2) / \delta$. Assuming additionally that min $c(x, y)-\lambda>$ $2 \kappa\left(M_{1}+M_{2}\right)$, we have

$\mathcal{L}_{\varepsilon} v_{\varepsilon}-\lambda v_{\varepsilon} \geq\left(-\kappa\left(M_{1}+\varepsilon M_{2}\right)+\left(c\left(x, x / \varepsilon^{\alpha}\right)-\lambda\right)\right) \exp (\kappa \gamma / \varepsilon)-\left(c\left(x, x / \varepsilon^{\alpha}\right)-\lambda\right)>0 \quad$ in $\Omega \backslash \Omega^{\prime}$.

Thus setting $\kappa:=\max \left\{\kappa_{1}, \kappa_{2}\right\}$ and $\Lambda:=2 \kappa\left(M_{1}+M_{2}\right)-\min c(x, y)$ we get (4.2).

Now note that $\lambda_{\varepsilon}$ is also the first eigenvalue (the eigenvalue with the maximal real part) of the adjoint operator $\mathcal{L}_{\varepsilon}^{*} u=\varepsilon^{2} \frac{\partial^{2}}{\partial x_{i} \partial x_{j}}\left(a^{i j} u\right)-\varepsilon \frac{\partial}{\partial x_{i}}\left(b^{i} u\right)+c u$, and the corresponding eigenfunction $u_{\varepsilon}^{*}$ can be chosen positive in $\Omega$. Therefore, if $\lambda_{\varepsilon}<-\Lambda$ then $\left(\mathcal{L}_{\varepsilon} v_{\varepsilon}-\lambda_{\varepsilon} v_{\varepsilon}\right) u_{\varepsilon}^{*}>0$ in $\Omega$ that contradicts the Fredholm theorem.

The following two results show that, up to extracting a subsequence, functions $W_{\varepsilon}$ converge uniformly on compacts in $\Omega$. For brevity introduce the notation

$$
d(x)=\operatorname{dist}(x, \partial \Omega) .
$$

Lemma 8. For every $\kappa>0$ there is a constant $C_{\kappa}$, independent of $\varepsilon$, such that

$$
\left|W_{\varepsilon}(x)-W_{\varepsilon}(z)\right| \leq C_{\kappa}(|x-z|+\varepsilon) \quad \text { when } x, z \in \Omega, \min \{d(x), d(z)\} \geq \kappa \varepsilon .
$$

Proof. Let $x_{0} \in \Omega, d\left(x_{0}\right) \geq \kappa \varepsilon$. Changing the variables $x \mapsto x_{0}+\varepsilon y$ in operator (1.1) we find that $v_{\varepsilon}=u_{\varepsilon}\left(x_{0}+\varepsilon y\right)$ satisfies the equation

$$
a_{\varepsilon}^{i j}(y) \frac{\partial^{2} v_{\varepsilon}}{\partial y_{i} \partial y_{j}}+b_{\varepsilon}^{j}(y) \frac{\partial v_{\varepsilon}}{\partial y_{j}}+\left(c_{\varepsilon}(y)-\lambda_{\varepsilon}\right) v_{\varepsilon}=0 \quad \text { when }|y|<\kappa .
$$


Note that $a_{\varepsilon}^{i j}, b_{\varepsilon}^{j}$ and $c_{\varepsilon}$ are uniformly bounded and $a_{\varepsilon}^{i j} \zeta^{i} \zeta^{j} \geq m|\zeta|^{2}>0 \forall \zeta \neq 0$. Therefore by Harnack's inequality (see, e.g., [27]) we have $v_{\varepsilon}(y) \leq C_{\kappa}^{\prime} v_{\varepsilon}\left(y^{\prime}\right)$ when $|y|,\left|y^{\prime}\right|<\kappa / 2$, where $C_{\kappa}^{\prime}>0$ is independent of $x_{0}$ and $\varepsilon$. Thus

$$
\left|W_{\varepsilon}(x)-W_{\varepsilon}(z)\right| \leq\left(\log C_{\kappa}^{\prime}\right) \varepsilon, \quad \text { if }|x-z|<\kappa \varepsilon / 2 \quad \text { and } \min \{d(x), d(z)\} \geq \kappa \varepsilon .
$$

Since $\partial \Omega$ is $C^{2}$-smooth and $\Omega$ is connected, every two points $x, z \in \Omega$ such that $d(x) \geq \kappa \varepsilon$, $d(z) \geq \kappa \varepsilon$ can be connected by a chain of segments $\left[x_{i}, x_{i+1}\right], i=\overline{1, n_{\varepsilon}}$ with $\left|x_{i+1}-x_{i}\right|<\kappa \varepsilon / 2$, $\operatorname{dist}\left(x_{i}, \partial \Omega\right) \geq \kappa \varepsilon$ and $n_{\varepsilon} \leq C_{\kappa}^{\prime \prime}(|x-z| / \varepsilon+1)$. (We assume that $\varepsilon$ is sufficiently small.) Then iterating (4.4) we obtain (4.3).

Lemma 9. Let $\phi(x) \in C^{2}(\bar{\Omega})$ then every maximum point $x_{\varepsilon}$ of the function $u_{\varepsilon}(x) e^{\phi(x) / \varepsilon}$ satisfies $d\left(x_{\varepsilon}\right) \geq \kappa \varepsilon$ with some $\kappa>0$ independent of $\varepsilon$.

Proof. Consider the function $v_{\varepsilon}(x)=u_{\varepsilon} e^{\left(\phi(x)-\rho_{\varepsilon}(x)\right) / \varepsilon}$, where $\rho_{\varepsilon}=2 d(x)-\beta d^{2}(x) / \varepsilon$ and $\beta$ is a positive parameter to be chosen later. Since $\mathcal{L}_{\varepsilon} u_{\varepsilon}-\lambda_{\varepsilon} u_{\varepsilon}=0$ we have

$$
0=\mathcal{L}_{\varepsilon}\left(e^{\left(-\phi(x)+\rho_{\varepsilon}(x)\right) / \varepsilon} v_{\varepsilon}\right)-\lambda_{\varepsilon} e^{\left(-\phi(x)+\rho_{\varepsilon}(x)\right) / \varepsilon} v_{\varepsilon}=e^{\left(-\phi(x)+\rho_{\varepsilon}(x)\right) / \varepsilon} \tilde{\mathcal{L}}_{\varepsilon} v_{\varepsilon}
$$

the operator $\tilde{\mathcal{L}}_{\varepsilon}$ being given by

$$
\tilde{\mathcal{L}}_{\varepsilon} v=\varepsilon^{2} a^{i j}\left(x, x / \varepsilon^{\alpha}\right) \frac{\partial^{2} v}{\partial x_{i} \partial x_{j}}+\varepsilon\left(b_{j}\left(x, x / \varepsilon^{\alpha}\right)-2 a^{i j} \frac{\partial}{\partial x_{i}}\left(\phi-\rho_{\varepsilon}\right)\right) \frac{\partial v}{\partial x_{j}}+\tilde{c}_{\varepsilon}(x) v,
$$

with

$$
\tilde{c}_{\varepsilon}(x)=c\left(x, x / \varepsilon^{\alpha}\right)-\lambda_{\varepsilon}+H\left(\nabla\left(\phi-\rho_{\varepsilon}\right), x, x / \varepsilon^{\alpha}\right)-\varepsilon a^{i j}\left(x, x / \varepsilon^{\alpha}\right) \frac{\partial^{2}}{\partial x_{i} \partial x_{j}}\left(\phi(x)-\rho_{\varepsilon}(x)\right) .
$$

The coefficient $\tilde{c}_{\varepsilon}(x)$ depends on the parameter $\beta$ through the derivatives of $\rho_{\varepsilon}$, and we can choose $\beta$ so large that $\tilde{c}_{\varepsilon}<0$ in $\Omega \backslash \Omega_{\varepsilon / \beta}$ for every $\varepsilon>0$, where $\Omega_{\varepsilon / \beta}=\{x \in \Omega ; d(x)>\varepsilon / \beta\}$. Indeed, $\left|\nabla \rho_{\varepsilon}\right| \leq 4$ when $d(x) \leq \varepsilon / \beta$ while $\lambda_{\varepsilon} \geq-\Lambda$ (cf. Lemma 7) and

$$
a^{i j}\left(x, x / \varepsilon^{\alpha}\right) \frac{\partial^{2} \rho_{\varepsilon}}{\partial x_{i} \partial x_{j}} \leq-\frac{2}{\varepsilon} \beta a^{i j}\left(x, x / \varepsilon^{\alpha}\right) \frac{\partial d(x)}{\partial x_{i}} \frac{\partial d(x)}{\partial x_{j}}+C \leq-2 m \beta / \varepsilon+C \quad \text { in } \Omega \backslash \Omega_{\varepsilon / \beta},
$$

where $C$ is independent of $\varepsilon$ and $\beta$. Thus there is $\beta>0$ such that $\tilde{c}_{\varepsilon}(x)<0$ in $\Omega \backslash \Omega_{\varepsilon / \beta}$. With this choice of $\beta$ we get by the maximum principle applied to the equation $\tilde{\mathcal{L}}_{\varepsilon} v_{\varepsilon}=0$, that $v_{\varepsilon} \leq$ $\max \left\{v_{\varepsilon}(x) ; x \in \partial \Omega_{\varepsilon / \beta}\right\}$ in $\Omega \backslash \Omega_{\varepsilon / \beta}$, or $u_{\varepsilon} e^{\phi(x) / \varepsilon} \leq e^{\left(\rho_{\varepsilon}(x)-\varepsilon / \beta\right) / \varepsilon} \max \left\{u_{\varepsilon}(x) e^{\phi(x) / \varepsilon} ; x \in \partial \Omega_{\varepsilon / \beta}\right\}$ when $d(x)<\varepsilon / \beta$. Since $\rho_{\varepsilon}(x)<\varepsilon / \beta$ when $d(x)<\varepsilon / \beta$, setting $\kappa=1 / \beta$ we get the desired bound.

Corollary 10. There is a constant $\kappa>0$ such that every maximum point $x_{\varepsilon}$ of $u_{\varepsilon}$ satisfies $d\left(x_{\varepsilon}\right) \geq \kappa \varepsilon$.

Proof. We simply apply Lemma 9 with $\phi \equiv 0$. 
Lemma 8 and Corollary 10 imply that we can extract a subsequence of functions $W_{\varepsilon}$ converging uniformly on every compact in $\Omega$ to a limit $W(x) \in C(\bar{\Omega})$. Moreover, by Lemma 8 the function $W$ is Lipschitz continuous and $\max \left\{\left|W_{\varepsilon}(x)-W(x)\right| ; x \in \Omega, d(x) \geq \kappa \varepsilon\right\} \rightarrow 0$ for every $\kappa>0$.

Corollary 11. Let $W_{\varepsilon}$ converge (along a subsequence) to a function $W$ uniformly on every compact in $\Omega$. Then, for every $\phi \in C^{2}(\bar{\Omega})$, we have

$$
\lim _{\varepsilon \rightarrow 0} \min \left\{W_{\varepsilon}(x)-\phi(x) ; x \in \bar{\Omega}\right\}=\min \{W(x)-\phi(x) ; x \in \bar{\Omega}\}
$$

Proof. We know that $\max \left\{\left|W_{\varepsilon}(x)-W(x)\right| ; x \in \Omega, d(x) \geq \kappa \varepsilon\right\} \rightarrow 0$ as $\varepsilon \rightarrow 0$ for every $\kappa>0$. On the other hand, by Lemma $9, \min \left\{W_{\varepsilon}(x)-\phi(x) ; x \in \bar{\Omega}\right\}$ is attained at a point located on the distance at least $\kappa_{0} \varepsilon$ from the boundary $\partial \Omega$ for some $\kappa_{0}>0$ independent of $\varepsilon$. This implies that $\liminf _{\varepsilon \rightarrow 0} \min \left\{W_{\varepsilon}(x)-\phi(x) ; x \in \bar{\Omega}\right\} \geq \min \{W(x)-\phi(x) ; x \in \bar{\Omega}\}$. The opposite inequality $\lim \sup _{\varepsilon \rightarrow 0} \min \left\{W_{\varepsilon}(x)-\phi(x) ; x \in \bar{\Omega}\right\} \leq \min \{W(x)-\phi(x) ; x \in \bar{\Omega}\}$ is an easy consequence of the uniform convergence of $W_{\varepsilon}$ to $W$ on compacts in $\Omega$.

\section{$5 \quad$ Vanishing viscosity limit}

This section is devoted to the proof of Theorem 1. According to the results of the previous section we can assume that, up to a subsequence,

$$
\lambda_{\varepsilon} \rightarrow \lambda
$$

and

$$
\max \left\{\left|W_{\varepsilon}(x)-W(x)\right| ; x \in \Omega, d(x) \geq \kappa \varepsilon\right\} \rightarrow 0 \forall \kappa>0,
$$

where $W$ is some Lipschitz continuous function. We are going to show that the pair $\lambda$ and $W$ is a solution of problem (1.3), (1.4).

We follow the same scheme for $\alpha>1, \alpha=1$ and $\alpha<1$.

For an arbitrary function $\phi \in C^{2}(\bar{\Omega})$, let $W-\phi$ attain strict minimum at a point $x_{0} \in \bar{\Omega}$. We construct test functions of the form

$$
\phi_{\varepsilon}=\tilde{\phi}_{\varepsilon}-\left|x-x_{0}\right|^{2},
$$

where $\tilde{\phi}_{\varepsilon} \rightarrow \phi$ uniformly in $\bar{\Omega}$. Moreover, we will chose functions $\tilde{\phi}_{\varepsilon}$ with uniformly bounded first derivatives and such that

$$
-\varepsilon a^{i j}\left(x_{\varepsilon}, x_{\varepsilon} / \varepsilon^{\alpha}\right) \frac{\partial^{2} \tilde{\phi}_{\varepsilon}}{\partial x_{i} \partial x_{j}}\left(x_{\varepsilon}\right)+H\left(\nabla \tilde{\phi}_{\varepsilon}\left(x_{\varepsilon}\right), x_{\varepsilon}, x_{\varepsilon} / \varepsilon^{\alpha}\right) \rightarrow \bar{H}\left(\nabla \phi\left(x_{0}\right), x_{0}\right)
$$

for every sequence of points $x_{\varepsilon} \in \Omega$ such that $x_{\varepsilon} \rightarrow x_{0}$. The existence of such functions $\tilde{\phi}_{\varepsilon}$ will be justified later on. 
Let us now show that minima points of functions $W_{\varepsilon}-\phi_{\varepsilon}$ converge to $x_{0}$. Indeed,

$$
\left|\min \left(W_{\varepsilon}-\phi_{\varepsilon}\right)-\min \left(W_{\varepsilon}-\phi+\left|x-x_{0}\right|^{2}\right)\right| \rightarrow 0
$$

since $\phi_{\varepsilon}(x) \rightarrow \phi(x)-\left|x-x_{0}\right|^{2}$ uniformly in $\bar{\Omega}$. If $x_{\varepsilon}$ is a minimum point of $W_{\varepsilon}-\phi_{\varepsilon}$ then in view of Corollary 11 we have

$$
\min \left(W_{\varepsilon}-\phi_{\varepsilon}\right)=W_{\varepsilon}\left(x_{\varepsilon}\right)-\phi\left(x_{\varepsilon}\right)+\left|x_{\varepsilon}-x_{0}\right|^{2}+\bar{o}(1) \geq \min (W-\phi)+\left|x_{\varepsilon}-x_{0}\right|^{2}+\bar{o}(1)
$$

On the other hand, choosing a sequence of points $\tilde{x}_{\varepsilon} \in \Omega$ such that $d\left(\tilde{x}_{\varepsilon}\right)=\left|\tilde{x}_{\varepsilon}-x_{0}\right|=\varepsilon$ we have

$$
\min \left(W_{\varepsilon}-\phi_{\varepsilon}\right) \leq W_{\varepsilon}\left(\tilde{x}_{\varepsilon}\right)-\phi_{\varepsilon}\left(\tilde{x}_{\varepsilon}\right) \rightarrow W\left(x_{0}\right)-\phi\left(x_{0}\right)=\min \left(W_{0}-\phi\right),
$$

therefore $x_{\varepsilon} \rightarrow x_{0}$.

Now, if $x_{\varepsilon}$ is a minimum point of $\left(W_{\varepsilon}-\phi_{\varepsilon}\right)$ then clearly $x_{\varepsilon} \in \Omega$, hence $\nabla \phi_{\varepsilon}\left(x_{\varepsilon}\right)=\nabla W_{\varepsilon}\left(x_{\varepsilon}\right)$ and $a^{i j}\left(x_{\varepsilon}, x_{\varepsilon} / \varepsilon^{\alpha}\right) \frac{\partial^{2} W_{\varepsilon}}{\partial x_{i} \partial x_{j}}\left(x_{\varepsilon}\right) \geq a^{i j}\left(x_{\varepsilon}, x_{\varepsilon} / \varepsilon^{\alpha}\right) \frac{\partial^{2} \phi_{\varepsilon}}{\partial x_{i} \partial x_{j}}\left(x_{\varepsilon}\right)$. We use (1.2) to get

$$
H\left(\nabla W_{\varepsilon}\left(x_{\varepsilon}\right), x_{\varepsilon}, x_{\varepsilon} / \varepsilon^{\alpha}\right)-\lambda_{\varepsilon} \geq \varepsilon a^{i j}\left(x_{\varepsilon}, x_{\varepsilon} / \varepsilon^{\alpha}\right) \frac{\partial^{2} \phi_{\varepsilon}}{\partial x_{i} \partial x_{j}}\left(x_{\varepsilon}\right)
$$

or

$$
-\varepsilon a^{i j}\left(x_{\varepsilon}, x_{\varepsilon} / \varepsilon^{\alpha}\right) \frac{\partial^{2} \tilde{\phi}_{\varepsilon}}{\partial x_{i} \partial x_{j}}\left(x_{\varepsilon}\right)+H\left(\nabla \tilde{\phi}_{\varepsilon}\left(x_{\varepsilon}\right), x_{\varepsilon}, x_{\varepsilon} / \varepsilon^{\alpha}\right)-\lambda_{\varepsilon}+\underline{O}\left(\varepsilon+\left|x_{\varepsilon}-x_{0}\right|\right) \geq 0 .
$$

Next we use (5.3) to pass to the limit in (5.4) as $\varepsilon \rightarrow 0$ that leads to the desired inequality

$$
\bar{H}\left(\nabla \phi\left(x_{0}\right), x_{0}\right) \geq \lambda
$$

If $W-\phi$ attains strict maximum at a point $x_{0} \in \Omega$, we argue similarly. We construct test functions of the form $\phi_{\varepsilon}=\tilde{\phi}_{\varepsilon}$ with $\tilde{\phi}_{\varepsilon}$ satisfying (5.3) and such that $\tilde{\phi}_{\varepsilon} \rightarrow \phi$ uniformly in $\bar{\Omega}$. Note that this time $x_{0} \notin \partial \Omega$ and therefore we can always chose a sequence of local maxima points $x_{\varepsilon}$ of $W_{\varepsilon}-\phi_{\varepsilon}$ converging to $x_{0}$. Then using the same arguments as in the proof of (5.5) we derive

$$
\bar{H}\left(\nabla \phi\left(x_{0}\right), x_{0}\right) \geq \lambda .
$$

Thus $W(x)$ is a viscosity solution of (1.3), (1.4).

It remains to construct functions $\tilde{\phi}_{\varepsilon}$ that have uniformly bounded first derivatives, satisfy (5.3) and converge to $\phi$ uniformly in $\bar{\Omega}$.

Case $\alpha>1$. We set

$$
\tilde{\phi}_{\varepsilon}(x)=\phi(x)+\varepsilon^{2 \alpha-1} \theta\left(x / \varepsilon^{\alpha}\right),
$$

where $\theta(y)$ is a $Y$-periodic solution of

$$
-a^{i j}\left(x_{0}, y\right) \frac{\partial^{2} \theta}{\partial y_{i} \partial y_{j}}=\bar{H}\left(\nabla \phi\left(x_{0}\right), x_{0}\right)-H\left(\nabla \phi\left(x_{0}\right), x_{0}, y\right)
$$


Thanks to (2.3) such a solution does exist, for (2.3) is nothing but the solvability condition for (5.7). Moreover, since the coefficients and the right hand side in (5.7) are Lipschitz continuous, $\theta \in C^{2,1}$ (see, e.g., [20]). Therefore if $x_{\varepsilon} \rightarrow x_{0}$ as $\varepsilon \rightarrow 0$, then we have

$$
\begin{aligned}
-\varepsilon a^{i j}\left(x_{\varepsilon}, x_{\varepsilon} / \varepsilon^{\alpha}\right) & \frac{\partial^{2} \tilde{\phi}_{\varepsilon}\left(x_{\varepsilon}\right)}{\partial x_{i} \partial x_{j}}+H\left(\nabla \tilde{\phi}_{\varepsilon}\left(x_{\varepsilon}\right), x_{\varepsilon}, x_{\varepsilon} / \varepsilon^{\alpha}\right)=-a^{i j}\left(x_{\varepsilon}, x_{\varepsilon} / \varepsilon^{\alpha}\right) \frac{\partial^{2} \theta\left(x_{\varepsilon} / \varepsilon^{\alpha}\right)}{\partial y_{i} \partial y_{j}}+\underline{O}(\varepsilon) \\
+ & H\left(\nabla \phi\left(x_{\varepsilon}\right)+\underline{O}\left(\varepsilon^{\alpha-1}\right), x_{\varepsilon}, x_{\varepsilon} / \varepsilon^{\alpha}\right)=-a^{i j}\left(x_{0}, x_{\varepsilon} / \varepsilon^{\alpha}\right) \frac{\partial^{2} \theta\left(x_{\varepsilon} / \varepsilon^{\alpha}\right)}{\partial y_{i} \partial y_{j}} \\
& +H\left(\nabla \phi\left(x_{0}\right), x_{0}, x_{\varepsilon} / \varepsilon^{\alpha}\right)+\underline{O}\left(\left|x-x_{\varepsilon}\right|+\varepsilon+\varepsilon^{\alpha-1}\right)=\bar{H}\left(\nabla \phi\left(x_{0}\right), x_{0}\right)+\bar{o}(1) .
\end{aligned}
$$

Case $\alpha=1$. Set $\tilde{\phi}_{\varepsilon}(x)=\phi(x)+\varepsilon \theta(x / \varepsilon)$, where $\theta(y)=-\log \vartheta(y)$ and $\vartheta(y)$ is the unique (up to multiplication by a positive constant) $Y$-periodic positive solution of

$$
\left.a^{i j}\left(x_{0}, y\right) \frac{\partial^{2} \vartheta}{\partial y_{i} \partial y_{j}}+\hat{b}^{j}(y) \frac{\partial \vartheta}{\partial y_{j}}+\hat{c}(y)\right) \vartheta=\bar{H}(p, x) \vartheta
$$

where $p=\nabla \phi\left(x_{0}\right), \hat{b}^{j}(y)=b^{j}\left(x_{0}, y\right)-2 a^{i j}\left(x_{0}, y\right) p_{i}, \hat{c}(y)=a^{i j}\left(x_{0}, y\right) p_{i} p_{j}-b^{j}\left(x_{0}, y\right) p_{j}+c\left(x_{0}, y\right)$. By a standard elliptic regularity result we have $\theta \in C^{2,1}$ (see [20]).

Simple calculations show that $\theta(y)$ satisfies

$$
-a^{i j}\left(x_{0}, y\right) \frac{\partial^{2} \theta}{\partial y_{i} \partial y_{j}}+H\left(\nabla \phi\left(x_{0}\right)+\nabla \theta, x_{0}, y\right)=\bar{H}\left(\nabla \phi\left(x_{0}\right), x_{0}\right)
$$

Then we easily conclude that

$$
\begin{aligned}
-\varepsilon a^{i j}\left(x_{\varepsilon}, x_{\varepsilon} / \varepsilon\right) & \frac{\partial^{2} \tilde{\phi}_{\varepsilon}\left(x_{\varepsilon}\right)}{\partial x_{i} \partial x_{j}}+H\left(\nabla \tilde{\phi}_{\varepsilon}\left(x_{\varepsilon}\right), x_{\varepsilon}, x_{\varepsilon} / \varepsilon\right)=-a^{i j}\left(x_{\varepsilon}, x_{\varepsilon} / \varepsilon\right) \frac{\partial^{2} \theta\left(x_{\varepsilon} / \varepsilon\right)}{\partial y_{i} \partial y_{j}}+\underline{O}(\varepsilon) \\
+ & H\left(\nabla \phi\left(x_{\varepsilon}\right)+\nabla \theta\left(x_{\varepsilon} / \varepsilon\right), x_{\varepsilon}, x_{\varepsilon} / \varepsilon\right)=-a^{i j}\left(x_{0}, x_{\varepsilon} / \varepsilon\right) \frac{\partial^{2} \theta\left(x_{\varepsilon} / \varepsilon\right)}{\partial y_{i} \partial y_{j}} \\
+ & H\left(\nabla \phi\left(x_{0}\right)+\nabla \theta\left(x_{\varepsilon} / \varepsilon\right), x_{0}, x_{\varepsilon} / \varepsilon\right)+\underline{O}\left(\left|x_{\varepsilon}-x_{0}\right|+\varepsilon\right)=\bar{H}\left(\nabla \phi\left(x_{0}\right), x_{0}\right)+\bar{o}(1),
\end{aligned}
$$

as soon as $x_{\varepsilon} \rightarrow x_{0}$ when $\varepsilon \rightarrow 0$.

Case $\alpha<1$. Set $\tilde{\phi}_{\varepsilon}(x)=\phi(x)+\varepsilon^{\alpha} \theta_{\varepsilon}\left(x / \varepsilon^{\alpha}\right)$ where $\theta_{\varepsilon}$ is a $Y$-periodic solution of the equation

$$
-\varepsilon^{1-\alpha} a^{i j}\left(x_{0}, y\right) \frac{\partial^{2} \theta_{\varepsilon}}{\partial y_{i} \partial y_{j}}+H\left(p+\nabla \theta_{\varepsilon}(y), x_{0}, y\right)=\bar{H}_{\varepsilon}\left(p, x_{0}\right) \text { with } p=\nabla \phi\left(x_{0}\right)
$$

Such a solution exists if $\bar{H}_{\varepsilon}\left(p, x_{0}\right)$ coincides the first eigenvalue $\mu_{\varepsilon}$ (eigenvalue with the maximal real part) of the spectral problem

$$
\begin{aligned}
& \varepsilon^{2(1-\alpha)} a^{i j}\left(x_{0}, y\right) \frac{\partial^{2} \vartheta_{\varepsilon}}{\partial y_{i} \partial y_{j}}+\varepsilon^{1-\alpha} \hat{b}^{j}(y) \frac{\partial \vartheta_{\varepsilon}}{\partial y_{j}}+\hat{c}(y) \vartheta_{\varepsilon}=\mu_{\varepsilon} \vartheta_{\varepsilon} \\
& \vartheta_{\varepsilon} \quad \text { is } Y \text {-periodic, }
\end{aligned}
$$


where $\hat{b}^{j}, \hat{c}$ are as in (5.8). According to the Krein-Rutman theorem $\mu_{\varepsilon}$ is a real and simple eigenvalue and the corresponding eigenfunction $\vartheta_{\varepsilon}$ can be chosen positive. Then a solution of (5.10) is given by $\theta_{\varepsilon}=-\varepsilon^{1-\alpha} \log \vartheta_{\varepsilon}$. We invoke now the results obtained in Section 3 ,

$$
\bar{H}_{\varepsilon}\left(p, x_{0}\right) \rightarrow \bar{H}\left(p, x_{0}\right)=\bar{H}\left(\nabla \phi\left(x_{0}\right), x_{0}\right)
$$

(where the limit $\bar{H}\left(p, x_{0}\right)$ is described in (2.5)),

$$
\left\|\partial^{2} \vartheta_{\varepsilon} / \partial y_{i} \partial y_{j}\right\|_{L^{\infty}} \leq C / \varepsilon^{1-\alpha}
$$

This allows us to show (5.3) similarly to other cases considered above,

$$
\begin{gathered}
-\varepsilon a^{i j}\left(x_{\varepsilon}, x_{\varepsilon} / \varepsilon^{\alpha}\right) \frac{\partial^{2} \tilde{\phi}_{\varepsilon}\left(x_{\varepsilon}\right)}{\partial x_{i} \partial x_{j}}+H\left(\nabla \tilde{\phi}_{\varepsilon}\left(x_{\varepsilon}\right), x_{\varepsilon}, x_{\varepsilon} / \varepsilon^{\alpha}\right)=-\varepsilon^{1-\alpha} a^{i j}\left(x_{\varepsilon}, x_{\varepsilon} / \varepsilon^{\alpha}\right) \frac{\partial^{2} \theta_{\varepsilon}\left(x_{\varepsilon} / \varepsilon^{\alpha}\right)}{\partial y_{i} \partial y_{j}}+\underline{O}(\varepsilon) \\
+H\left(\nabla \phi\left(x_{\varepsilon}\right)+\nabla \theta_{\varepsilon}\left(x_{\varepsilon} / \varepsilon^{\alpha}\right), x_{\varepsilon}, x_{\varepsilon} / \varepsilon^{\alpha}\right)=-\varepsilon^{1-\alpha} a^{i j}\left(x_{0}, x_{\varepsilon} / \varepsilon^{\alpha}\right) \frac{\partial^{2} \theta_{\varepsilon}\left(x_{\varepsilon} / \varepsilon^{\alpha}\right)}{\partial y_{i} \partial y_{j}} \\
+H\left(\nabla \phi\left(x_{0}\right)+\nabla \theta_{\varepsilon}\left(x_{\varepsilon} / \varepsilon^{\alpha}\right), x_{0}, x_{\varepsilon} / \varepsilon^{\alpha}\right)+\underline{O}\left(\left|x-x_{\varepsilon}\right|+\varepsilon\right)=\bar{H}\left(\nabla \phi\left(x_{0}\right), x_{0}\right)+\bar{o}(1) .
\end{gathered}
$$

Theorem 1 is completely proved.

\section{Lower bound for eigenvalues via blow up analysis}

From now on we consider in details the special case when $\alpha=1$ and $c(x, y)=0$. Under the assumption (2.17) the eigenvalues $\lambda_{\varepsilon}$ of (1.1) converge to zero as $\varepsilon \rightarrow 0$. We are interested in the more precise (up to the order $\varepsilon$ ) asymptotics for $\lambda_{\varepsilon}$. We resolve this question by local analysis near points $\xi$ of the Aubry set $\mathcal{A}_{\bar{H}}$ of the effective Hamiltonian.

Fix a point $\xi \in \mathcal{A}_{\bar{H}}$.

Applying the maximum principle we see that $\lambda_{\varepsilon}<0$. On the other hand, it is well known that the eigenvalue $\lambda_{\varepsilon}$ is given by

$$
\lambda_{\varepsilon}=\inf \left\{\sup _{x \in \Omega} \frac{\mathcal{L}_{\varepsilon} \phi}{\phi} ; \phi \in C^{2}(\Omega) \cap C(\bar{\Omega}), \phi>0 \text { in } \Omega, \phi=0 \text { on } \partial \Omega\right\} .
$$

Therefore, for every given $\delta>0$, we have

$$
\lambda_{\varepsilon} \geq \varepsilon \tilde{\sigma}_{\varepsilon}
$$

where $\tilde{\sigma}_{\varepsilon}<0$ is the first eigenvalue of the equation $\mathcal{L}_{\varepsilon} v_{\varepsilon}-\delta|x-\xi|^{2} v_{\varepsilon}=\varepsilon \tilde{\sigma}_{\varepsilon} v_{\varepsilon}$ or

$$
\varepsilon a^{i j}(x, x / \varepsilon) \frac{\partial^{2} v_{\varepsilon}}{\partial x_{i} \partial x_{j}}+b^{j}(x, x / \varepsilon) \frac{\partial v_{\varepsilon}}{\partial x_{j}}-\frac{\delta|x-\xi|^{2}}{\varepsilon} v_{\varepsilon}=\tilde{\sigma}_{\varepsilon} v_{\varepsilon} \quad \text { in } \Omega
$$

with the Dirichlet condition $v_{\varepsilon}=0$ on $\partial \Omega$. We assume hereafter that the first eigenfunction $v_{\varepsilon}$ is normalized by $v_{\varepsilon}(\xi)=1$. 
Let us transform (6.2) to a form more convenient for the analysis. First, after changing variables $z=(x-\xi) / \sqrt{\varepsilon}$ and setting $w_{\varepsilon}(z)=v_{\varepsilon}(\xi+\sqrt{\varepsilon} z)$ equation (6.2) becomes

$$
a_{\xi, \xi / \varepsilon}^{i j}(\sqrt{\varepsilon} z, z / \sqrt{\varepsilon}) \frac{\partial^{2} w_{\varepsilon}}{\partial z_{i} z_{j}}+\frac{b_{\xi, \xi / \varepsilon}^{j}(\sqrt{\varepsilon} z, z / \sqrt{\varepsilon})}{\sqrt{\varepsilon}} \frac{\partial w_{\varepsilon}}{\partial z_{j}}-\delta|z|^{2} w_{\varepsilon}=\tilde{\sigma}_{\varepsilon} w_{\varepsilon} \quad \text { in }(\Omega-\xi) / \sqrt{\varepsilon} .
$$

Here (and below) the subscript " $\xi, \xi / \varepsilon$ " denotes the shift (translation) by $\xi$ in $x$ and by $\xi / \varepsilon$ in $y$, i.e., for instance, $a_{\xi, \xi / \varepsilon}^{i j}(x, y)=a_{\xi, \xi / \varepsilon}^{i j}(\xi+x, \xi / \varepsilon+y)$. Next multiply (6.3) by $\theta_{\xi, \xi / \varepsilon}^{*}(\sqrt{\varepsilon} z, z / \sqrt{\varepsilon})$, $\theta^{*}(x, y)$ being given by (2.16), to find after simple rearrengements

$$
\begin{aligned}
\frac{\partial}{\partial z_{i}}\left(\theta_{\xi, \xi / \varepsilon}^{*}(\sqrt{\varepsilon} z, z / \sqrt{\varepsilon}) a_{\xi, \xi / \varepsilon}^{i j}(\sqrt{\varepsilon} z, z / \sqrt{\varepsilon}) \frac{\partial w_{\varepsilon}}{\partial z_{j}}\right)+\frac{S_{\xi, \xi / \varepsilon}^{j}(\sqrt{\varepsilon} z, z / \sqrt{\varepsilon})}{\sqrt{\varepsilon}} \frac{\partial w_{\varepsilon}}{\partial z_{j}} \\
+\left(\frac{\bar{b}^{j}(\sqrt{\varepsilon} z+\xi)}{\sqrt{\varepsilon}}+\sqrt{\varepsilon} h_{\varepsilon}^{j}(z)\right) \frac{\partial w_{\varepsilon}}{\partial z_{j}}=\left(\tilde{\sigma}_{\varepsilon}+\delta|z|^{2}\right) \theta_{\xi, \xi / \varepsilon}^{*}(\sqrt{\varepsilon} z, z / \sqrt{\varepsilon}) w_{\varepsilon}
\end{aligned}
$$

where $S_{\xi, \xi / \varepsilon}^{j}(x, y)$ is obtained by shifts (as described above) from

$$
S^{j}(x, y)=b^{j}(x, y) \theta^{*}(x, y)-\frac{\partial}{\partial y_{i}}\left(a^{i j}(x, y) \theta^{*}(x, y)\right)-\bar{b}^{j}(x),
$$

and $h_{\varepsilon}^{j}$ are uniformly bounded functions. Since $\theta^{*}$ solves (2.16), the $Y$-periodic vector field $S(x, y)=\left(S^{1}(x, y), \ldots, S^{N}(x, y)\right)$ is divergence free, for every fixed $x$, and (due to the definition of $\bar{b})$ this field has zero mean over the period. Therefore we can find the representation

$$
S^{j}(x, y)=\frac{\partial}{\partial y_{i}} T^{i j}(x, y) \text { with } Y \text {-periodic in } y \text { skew-symmetric } T^{i j}(x, y)\left(T^{i j}=-T^{j i}\right) .
$$

Moreover, functions $T^{i j}$ are (can be chosen) continuous with bounded derivatives $\partial T^{i j} / \partial x_{k}$. We can thus rewrite (6.4) as

$\frac{\partial}{\partial z_{i}}\left(q_{\xi, \xi / \varepsilon}^{i j}(\sqrt{\varepsilon} z, z / \sqrt{\varepsilon}) \frac{\partial w_{\varepsilon}}{\partial z_{j}}\right)+\left(\frac{\bar{b}^{j}(\sqrt{\varepsilon} z+\xi)}{\sqrt{\varepsilon}}+\sqrt{\varepsilon} \tilde{h}_{\varepsilon}^{j}(z)\right) \frac{\partial w_{\varepsilon}}{\partial z_{j}}=\left(\tilde{\sigma}_{\varepsilon}+\delta|z|^{2}\right) \theta_{\xi, \xi / \varepsilon}^{*}(\sqrt{\varepsilon} z, z / \sqrt{\varepsilon}) w_{\varepsilon}$

where $q_{\xi, \xi / \varepsilon}^{i j}(x, y)=q^{i j}(\xi+x, \xi / \varepsilon+y), q^{i j}(x, y)=\theta^{*}(x, y) a^{i j}(x, y)+T^{i j}(x, y)$, and $\tilde{h}_{\varepsilon}^{j}$ are uniformly bounded functions. Note that on every fixed compact we have

$$
\frac{\bar{b}^{j}(\sqrt{\varepsilon} z+\xi)}{\sqrt{\varepsilon}} \rightarrow z_{i} \frac{\partial \bar{b}^{j}}{\partial x_{i}}(\xi)
$$

uniformly in $z$ as $\varepsilon \rightarrow 0$.

Lemma 12. If $\bar{b}(\xi)=0$ for some $\xi \in \bar{\Omega}$ then the first eigenvalue $\lambda_{\varepsilon}$ of the operator (2.14) satisfies the bound $-\Lambda \varepsilon \leq \lambda_{\varepsilon}<0$ with some $\Lambda>0$ independent of $\varepsilon$.

Proof. We know that $\lambda_{\varepsilon}<0$ and in the proof of the lower bound we assume first $\xi \in \Omega$. Then (6.5) holds in $B_{2}=\{z ;|z|<2\}$ for sufficiently small $\varepsilon$. Let us write (6.5) in the operator form $\mathcal{L}_{\varepsilon}^{(a u x)} w_{\varepsilon}=\tilde{\sigma}_{\varepsilon} \theta_{\xi, \xi / \varepsilon}^{*}(\sqrt{\varepsilon} z, z / \sqrt{\varepsilon}) w_{\varepsilon}$ and consider the parabolic equation for the operator $\mathcal{L}_{\varepsilon}^{(\text {aux })}$

$$
\frac{\partial \tilde{w}_{\varepsilon}}{\partial t}-\mathcal{L}_{\varepsilon}^{(\text {aux })} \tilde{w}_{\varepsilon}=0 \quad \text { in }(0,+\infty) \times B_{2}
$$


subject to the initial condition $\tilde{w}_{\varepsilon}(0, z)=w_{\varepsilon}(z)$ and the boundary condition $\tilde{w}_{\varepsilon}(t, z)=0$ on $(0,+\infty) \times \partial \Omega$. The solution $\tilde{w}_{\varepsilon}$ of this problem has the pointwise bound $\tilde{w}_{\varepsilon}(t, z) \leq$ $\exp \left(\tilde{\sigma}_{\varepsilon}\left(\min \theta^{*}\right) t\right) w_{\varepsilon}(z)$. This follows by the maximum principle applied to

$$
\left(\frac{\partial}{\partial t}-\mathcal{L}_{\varepsilon}^{(a u x)}\right)\left(\exp \left(\tilde{\sigma}_{\varepsilon}\left(\min \theta^{*}\right) t\right) w_{\varepsilon}(z)-\tilde{w}_{\varepsilon}(t, z)\right)=\tilde{\sigma}_{\varepsilon}\left(\min \theta^{*}-\theta_{\xi, \xi / \varepsilon}^{*}(\sqrt{\varepsilon} z, z / \sqrt{\varepsilon})\right) w_{\varepsilon}(z) \geq 0 .
$$

On the other hand, since the coefficients of the operator $\mathcal{L}_{\varepsilon}$ are uniformly bounded and the uniform ellipticity bound $\theta_{\xi, \xi / \varepsilon}^{*}(\sqrt{\varepsilon} z, z / \sqrt{\varepsilon}) a_{\xi, \xi / \varepsilon}^{i j}(\sqrt{\varepsilon} z, z / \sqrt{\varepsilon}) \zeta^{i} \zeta^{j} \geq\left(\min \theta^{*}\right) m|\zeta|^{2}$ holds, by the Aronson estimate (see [3]) we have

$$
\min \left\{\tilde{w}_{\varepsilon}(1, z) ; z \in B_{1}\right\} \geq M \min \left\{\tilde{w}_{\varepsilon}(0, z) ; z \in B_{1}\right\}
$$

with $M>0$ independent of $\varepsilon$, where $B_{1}$ is the unit ball $B_{1}=\{z ;|z|<1\}$. This yields

$$
e^{\left(\min \theta^{*}\right) \tilde{\sigma}_{\varepsilon}} \min _{B_{1}} w_{\varepsilon} \geq \min \left\{\tilde{w}_{\varepsilon}(1, z) ; z \in B_{1}\right\} \geq M \min \left\{\tilde{w}_{\varepsilon}(0, z) ; z \in B_{1}\right\}=M \min _{B_{1}} w_{\varepsilon}
$$

i.e. $\tilde{\sigma}_{\varepsilon} \geq \log M / \min \theta^{*}=:-\Lambda$. Thus $\tilde{\sigma}_{\varepsilon} \geq-\Lambda$ and $\lambda_{\varepsilon} \geq-\Lambda \varepsilon$.

Finally, in the case $\xi \in \partial \Omega$ we can repeat the above argument taking $\xi_{\varepsilon} \in \Omega$ in place of $\xi$, with $\left|\xi_{\varepsilon}-\xi\right|=\operatorname{dist}\left(\xi_{\varepsilon}, \partial \Omega\right)=2 \sqrt{\varepsilon}$.

In the proof of Lemma 12 we have got a uniform lower bound for $\tilde{\sigma}_{\varepsilon}$ which (in conjunction with the obvious inequality $\tilde{\sigma}_{\varepsilon}<0$ ) allows one to obtain uniform bounds for the norm of $w_{\varepsilon}$ in $C^{0, \beta}(K)$ (with $\beta>0$ depending only on bounds for coefficients in (6.5)) and $H^{1}(K)$, for every compact $K$ (see, e.g., [20]). Thus, up to extracting a subsequence, $w_{\varepsilon} \rightarrow w$ in $C_{\text {loc }}\left(\mathbb{R}^{N}\right)$ and $\tilde{\sigma}_{\varepsilon} \rightarrow \tilde{\sigma}$. Moreover, using well established homogenization technique based on the div-curl Lemma we get that $w$ solves

$$
Q^{i j} \frac{\partial^{2} w}{\partial z_{i} \partial z_{j}}+B^{i j} z_{i} \frac{\partial w}{\partial z_{j}}-\delta|z|^{2} w=\tilde{\sigma} w \quad \text { in } \mathbb{R}^{N}
$$

where $Q^{i j}=Q^{j i}$ are some constant coefficients satisfying the ellipticity condition (actually, one can check that $\left.Q^{i j}=\frac{1}{2} \frac{\partial^{2} \bar{H}}{\partial p_{i} \partial p_{j}}(0, \xi)\right)$ and

$$
B^{i j}\left(=B^{i j}(\xi)\right)=\frac{\partial \bar{b}^{j}}{\partial x_{i}}(\xi)
$$

Since we assumed the normalization $w_{\varepsilon}(0)=1$, we see that $w(z)$ is a nontrivial solution of (6.6). Moreover, if $z_{\varepsilon}$ is a maximum point of $w_{\varepsilon}(z)$ we get from (6.3) $\left|z_{\varepsilon}\right|^{2} \leq-\tilde{\sigma}_{\varepsilon} / \delta$ therefore, thanks to Lemma 12, $\left|z_{\varepsilon}\right| \leq C$. It follows that $w(z)$ is a bounded positive solution of (66.6). A solution of (6.6) can be constructed in the form $w(z)=e^{-\Gamma_{\delta}^{i j} z_{i} z_{j}}$ with a symmetric positive definite matrix $\left(\Gamma_{\delta}^{i j}\right)_{i, j=\overline{1, N}}$. Indeed, consider the following matrix Riccati equation

$$
4 \Gamma_{\delta} Q \Gamma_{\delta}-B \Gamma_{\delta}-\Gamma_{\delta} B^{*}-\delta I=0
$$


where $I$ denotes the unit matrix. It is well-known that there exists a unique positive definite solution $\Gamma_{\delta}$ (since $\delta>0$ and $Q$ is positive definite, see [28]). Then $w(z)=e^{-\Gamma_{\delta}^{i j} z_{i} z_{j}}$ is a positive bounded solution of ([6.6) corresponding to the eigenvalue $\tilde{\sigma}=-2 \operatorname{tr}\left(Q \Gamma_{\delta}\right)$. Next observe that by means of the gauge transformation $\tilde{w}(z)=e^{-r|z|^{2}} w(z)(r>0)$ equation (6.6) is reduced to $Q^{i j} \frac{\partial^{2} \tilde{w}}{\partial z_{i} \partial z_{j}}+\left(B^{i j}+4 r Q^{i j}\right) z_{i} \frac{\partial \tilde{w}}{\partial z_{j}}+\left(4 r^{2} Q^{i j} z_{i} z_{j}+2 r \operatorname{tr} Q+2 r B^{i j} z_{i} z_{j}-\delta|z|^{2}\right) \tilde{w}=\tilde{\sigma} \tilde{w} \quad$ in $\mathbb{R}^{N}$.

For sufficiently small $r>0$ we have $\left(\left(4 r^{2} Q^{i j} z_{i} z_{j}+2 r \operatorname{tr} Q+2 r B^{i j} z_{i} z_{j}-\delta|z|^{2}\right) \rightarrow-\infty\right.$ and $\tilde{w}(z) \rightarrow 0$ as $|z| \rightarrow \infty$. Then, according to [37], the eigenvalue $\tilde{\sigma}$ corresponding to such a positive eigenfunction $\tilde{w}$ (vanishing as $|z| \rightarrow \infty)$ is unique. Thus $\tilde{\sigma}=-2 \operatorname{tr}\left(Q \Gamma_{\delta}\right)$, and summarizing the above analysis we have $\liminf _{\varepsilon \rightarrow 0} \lambda_{\varepsilon} / \varepsilon \geq-2 \operatorname{tr}\left(Q \Gamma_{\delta}\right)$. Finally note that $\Gamma_{\delta}$ converges to the maximal positive semi-definite solution of the Bernoulli equation (see, e.g., [28])

$$
4 \Gamma Q \Gamma-B \Gamma-\Gamma B^{*}=0,
$$

as $\delta \rightarrow+0$. Calculations presented in Appendix $\mathrm{C}$ show that $-2 \operatorname{tr}(Q \Gamma)=\sigma(\xi)$ with $\sigma(\xi)$ being the sum of negative real parts of the eigenvalues of $-B(\xi)$. Thus, after maximizing in $\xi \in \mathcal{A}_{\bar{H}}$ we get

$$
\liminf _{\varepsilon \rightarrow 0} \lambda_{\varepsilon} / \varepsilon \geq \bar{\sigma}=\max \left\{\sigma(\xi) ; \xi \in \mathcal{A}_{\bar{H}}\right\}
$$

\section{Upper bound for eigenvalues and selection of the additive eigenfunction}

In this section we derive an upper bound for eigenvalues which completes the proof of the asymptotic expansion formula (2.18). Similarly to the previous section we make use of the blow up analysis near points of the Aubry set. We consider here only special (so-called significant) points of the Aubry set, where we can control the asymptotic behavior of rescaled eigenfunctions at infinity. We will show that only these special points matter to the leading term of eigenvalues and eigenfunctions.

Due to Theorem 1, up to extracting a subsequence, the functions $W_{\varepsilon}=-\varepsilon \log u_{\varepsilon}$ converge uniformly on compacts to a viscosity solution $W$ of $\bar{H}(\nabla W(x), x)=0$ in $\Omega, \bar{H}(\nabla W(x), x) \geq 0$ on $\partial \Omega$. It follows that $W$ has the representation $W(x)=\min \left\{d_{\bar{H}}(x, \xi)+W(\xi) ; \xi \in \mathcal{A}_{\bar{H}}\right\}$.

We will say that point $\xi \in \mathcal{A}_{\bar{H}}$ is significant if

$$
W(x)=d_{\bar{H}}(x, \xi)+W(\xi) \quad \text { in a neighborhood of } \xi
$$

Otherwise we call $\xi$ negligible. For every negligible point $\xi \in \mathcal{A}_{\bar{H}}$ there are sequences $x^{k} \rightarrow \xi$ and $\xi^{k} \in \mathcal{A}_{\bar{H}} \backslash\{\xi\}$ such that $d_{\bar{H}}\left(x^{k}, \xi\right)+W(\xi)>d_{\bar{H}}\left(x^{k}, \xi^{k}\right)+W\left(\xi^{k}\right)$. Passing to the limit (possibly along a subsequence) and using the continuity of the distance function we get $d_{\bar{H}}\left(\xi, \xi^{\prime}\right)=$ 
$W(\xi)-W\left(\xi^{\prime}\right)$ for some $\xi^{\prime} \in \mathcal{A}_{\bar{H}}, \xi^{\prime} \neq \xi$ (we always have $d_{\bar{H}}\left(\xi, \xi^{\prime}\right) \geq W(\xi)-W\left(\xi^{\prime}\right)$ ). Now let us introduce the (partial) order relation $\preceq$ on $\mathcal{A}_{\bar{H}}$ by setting

$$
\xi^{\prime} \preceq \xi \Longleftrightarrow d_{\bar{H}}\left(\xi, \xi^{\prime}\right)=W(\xi)-W\left(\xi^{\prime}\right) .
$$

This relation is clearly reflexive, its transitivity is a consequence of the triangle inequality $d_{\bar{H}}\left(\xi, \xi^{\prime \prime}\right) \leq d_{\bar{H}}\left(\xi, \xi^{\prime}\right)+d_{\bar{H}}\left(\xi^{\prime}, \xi^{\prime \prime}\right)$ while the antisymmetry follows from the inequality $S_{\bar{H}}\left(\xi, \xi^{\prime}\right)>$ 0 held for all $\xi, \xi^{\prime} \in \mathcal{A}_{\bar{H}}$ with $\xi \neq \xi^{\prime}$. Then we see that every minimal element $\xi \in \mathcal{A}_{\bar{H}}$ is a significant point. Since $\mathcal{A}_{\bar{H}}$ is finite there exists a minimal element, i.e. there is at least one significant point $\xi \in \mathcal{A}_{\bar{H}}$.

Let us fix a significant point $\xi \in \mathcal{A}_{\bar{H}}$. From now on we assume that $u_{\varepsilon}$ is normalized by $u_{\varepsilon}(\xi)=1$, unless otherwise is specified; the $W$ will also refer to the limit of scaled logarithmic transformations of $u_{\varepsilon}$ normalized in this way. Thanks to the upper and lower bounds for the eigenvalue $\lambda_{\varepsilon}$ we have $\lambda_{\varepsilon} / \varepsilon \rightarrow \sigma$ as $\varepsilon \rightarrow 0$ along a subsequence. Then we argue exactly as in the proof of the lower bound for $\lambda_{\varepsilon}$. We consider rescaled eigenfunctions $w_{\varepsilon}(z)=u_{\varepsilon}(\xi+\sqrt{\varepsilon} z)$ that are solutions of

$\frac{\partial}{\partial z_{i}}\left(q_{\xi, \frac{\xi}{\varepsilon}}^{i j}(\sqrt{\varepsilon} z, z / \sqrt{\varepsilon}) \frac{\partial w_{\varepsilon}}{\partial z_{j}}\right)+\left(\frac{\bar{b}^{j}(\sqrt{\varepsilon} z+\xi)}{\sqrt{\varepsilon}}+\sqrt{\varepsilon} \tilde{h}_{\varepsilon}^{j}(z)\right) \frac{\partial w_{\varepsilon}}{\partial z_{j}}=\frac{\lambda_{\varepsilon}}{\varepsilon} \theta_{\xi, \frac{\xi}{\varepsilon}}^{*}(\sqrt{\varepsilon} z, z / \sqrt{\varepsilon}) w_{\varepsilon} \quad$ in $\frac{\Omega-\xi}{\sqrt{\varepsilon}}$.

Up to extracting a further subsequence, they converge in $C(K)$ and weakly in $H^{1}(K)$ (for every compact $K$ ) to a positive solution of

$$
Q^{i j} \frac{\partial^{2} w}{\partial z_{i} \partial z_{j}}+B^{i j} z_{i} \frac{\partial w}{\partial z_{j}}=\sigma w \quad \text { in } \mathbb{R}^{N} .
$$

Eigenvalue problem (7.2) possesses, in general, many solutions even in the class of positive eigenfunctions $w(z)$. We will uniquely identify $\sigma$ studying the asymptotic behavior of $w(z)$ as $|z| \rightarrow \infty$. More precisely, we will show that

$$
w(z) e^{\mu\left|\Pi_{s}^{*} z\right|^{2}-\nu\left|\Pi_{u}^{*} z\right|^{2}} \text { is bounded on } \mathbb{R}^{N} \text { for some } \mu>0 \text { and every } \nu>0,
$$

where $\Pi_{s}$ and $\Pi_{u}$ denote spectral projectors on the invariant subspaces of the matrix $B$ corresponding to the eigenvalues with positive and negative real parts (stable and unstable subspaces of the system $\dot{z}_{i}=-B^{i j} z_{j}$ ). This allows to use the following uniqueness result.

Lemma 13. Let $w(z)$ be a positive solution of (7.2) satisfying (7.3). Then $w(z)=C e^{-\Gamma^{i j} z_{i} z_{j}}$ with $C>0$, where $\Gamma$ is the maximal positive semi-definite solution of (6.7). Moreover, we have $\sigma=-2 \operatorname{tr}(\Gamma Q)$.

Proof. First observe that $w(z)=C e^{-\Gamma^{i j} z_{i} z_{j}}$ satisfies (7.3). This follows from the relation $\Gamma=$ $\Pi_{s} \Gamma \Pi_{s}^{*} \geq \gamma \Pi_{s} \Pi_{s}^{*}$ where the inequality holds for some $\gamma>0$ in the sense of quadratic forms, see Proposition 17 in Appendix [C, It is also clear that $w(z)$ does solve (7.2) with $\sigma=-2 \operatorname{tr}(\Gamma Q)$. 
To justify the uniqueness of $\sigma$ and $w(z)$ we make use of a gauge transformation $\tilde{w}(z)=$ $e^{\phi(z)} w(z)$, with a quadratic function $\phi(z)$ to be constructed later on, which leads to the equation of the form

$$
Q^{i j} \frac{\partial^{2} \tilde{w}}{\partial z_{i} \partial z_{j}}+\tilde{B}^{i j} z_{i} \frac{\partial \tilde{w}}{\partial z_{j}}+\tilde{C}(z) \tilde{w}=\sigma \tilde{w} \quad \text { in } \mathbb{R}^{N} .
$$

We will choose $\phi(z)$ so that $\tilde{C}(z) \rightarrow-\infty, \tilde{w}(z) \rightarrow 0$ as $|z| \rightarrow \infty$. Then, by [37], there is a unique $\sigma$ such that (17.4) has a positive solution $\tilde{w}(z)$ vanishing as $|z| \rightarrow \infty(\tilde{w}(z)$ is also unique up to multiplication by a positive constant).

Construct $\phi(z)$ by setting $\phi=r A_{s}^{i j} z_{i} z_{j}-r A_{u}^{i j} z_{i} z_{j}$, with symmetric matrices $A_{s}$ and $A_{u}$, to get $\tilde{B}^{i j}=B^{i j}+4 r Q^{j l}\left(A_{u}^{l i}-A_{s}^{l i}\right)$ and

$$
\begin{aligned}
\tilde{C}(z)=4 r^{2}\left(A_{u}^{i l}-A_{s}^{i l}\right) Q^{l m}( & \left.A_{u}^{m j}-A_{s}^{m j}\right) z_{i} z_{j} \\
& +r\left(\left(B^{i l}\left(A_{u}^{l j}-A_{s}^{l j}\right)+\left(A_{u}^{i l}-A_{s}^{i l}\right) B^{l j}\right) z_{i} z_{j}+2 \operatorname{tr}\left(Q\left(A_{u}-A_{s}\right)\right)\right) .
\end{aligned}
$$

Define $A_{s}$ and $A_{u}$ as solutions of the Lyapunov matrix equations

$$
B A_{s}+A_{s} B^{*}=\Pi_{s} \Pi_{s}^{*}, B A_{u}+A_{u} B^{*}=-\Pi_{u} \Pi_{u}^{*}
$$

given by

$$
A_{s}=\int_{-\infty}^{0} e^{B t} \Pi_{s} \Pi_{s}^{*} e^{B^{*} t} \mathrm{~d} t, \quad A_{u}=\int_{0}^{\infty} e^{B t} \Pi_{u} \Pi_{u}^{*} e^{B^{*} t} \mathrm{~d} t,
$$

and choose sufficiently small $r_{0}>0$ in such a way that the matrix

$4 r\left(A_{u}-A_{s}\right) Q\left(A_{u}-A_{s}\right)+B\left(A_{u}-A_{s}\right)+\left(A_{u}-A_{s}\right) B^{*}=4 r\left(A_{u}-A_{s}\right) Q\left(A_{u}-A_{s}\right)-\Pi_{s} \Pi_{s}^{*}-\Pi_{u} \Pi_{u}^{*}$

is negative definite for $0<r<r_{0}$. Then $\tilde{C}(z) \rightarrow-\infty$ as $|z| \rightarrow \infty$. It remains to see that if $w(z)$ satisfies (7.3) then choosing small enough $r>0$ we have $\tilde{w}(z) \rightarrow 0$ as $|z| \rightarrow \infty$. Here we have used the inequalities $A_{s} \leq \gamma_{1} \Pi_{s} \Pi_{s}^{*}$ and $A_{u} \geq \gamma_{2} \Pi_{u} \Pi_{u}^{*}$ for some $\gamma_{1}, \gamma_{2}>0$.

So far we know that $\lambda_{\varepsilon} / \varepsilon \rightarrow \sigma$ and $w_{\varepsilon}(z)=u_{\varepsilon}(\xi+\sqrt{\varepsilon} z)$ converge in uniformly on compacts to a positive solution of (7.2). In order to apply Lemma 13 we need only to show (17.3). To this end we first construct a quadratic function $\Phi_{\mu}^{\nu}(x)$ satisfying

$$
\bar{H}\left(\nabla \Phi_{\mu}^{\nu}(x), x\right) \leq-\delta|x-\xi|^{2} \quad \text { in a neighborhood } U(\xi) \text { of } \xi
$$

for some $\delta>0$.

Lemma 14. Let us set $\phi_{s}(x):=A_{s}^{i j} x_{i} x_{j}$ and $\phi_{u}(x):=A_{u}^{i j} x_{i} x_{j}$, where $A_{s}$ and $A_{u}$ are solutions of the Lyapunov matrix equation (7.5) given by (7.6). Then the function

$$
\Phi_{\mu}^{\nu}(x):=\mu \phi_{s}(x-\xi)-\nu \phi_{u}(x-\xi)
$$

satisfies (7.7) for some $\delta>0$, provided that $0<\mu, \nu<r$ and $r>0$ is sufficiently small. 
Proof. We have, as $x \rightarrow \xi$,

$$
\begin{aligned}
& \bar{H}\left(\nabla \Phi_{\mu}^{\nu}(x), x\right) \leq \bar{H}(0, x)+ \frac{\partial \bar{H}}{\partial p_{j}}(0, x) \frac{\partial \Phi_{\mu}^{\nu}}{\partial x_{j}}(x)+C\left|\nabla \Phi_{\mu}^{\nu}(x)\right|^{2} \\
&=-\left(x_{i}-\xi_{i}\right) \frac{\partial \bar{b}^{j}}{\partial x_{i}}(\xi) \frac{\partial \Phi_{\mu}^{\nu}}{\partial x_{j}}(x)+C\left|\nabla \Phi_{\mu}^{\nu}(x)\right|^{2}+\bar{o}\left(|x-\xi|^{2}\right) \\
& \leq-2\left(x_{i}-\xi_{i}\right) B^{i j}\left(\mu A_{s}^{j l}-\nu A_{u}^{j l}\right)\left(x_{l}-\xi_{l}\right) \\
& \quad+C_{1}\left(\mu^{2}\left|\Pi_{s}^{*}(x-\xi)\right|^{2}+\nu^{2}\left|\Pi_{u}^{*}(x-\xi)\right|^{2}\right)+\bar{o}\left(|x-\xi|^{2}\right) .
\end{aligned}
$$

Note that $B\left(\mu A_{s}-\nu A_{u}\right)+\left(\mu A_{s}-\nu A_{u}\right) B^{*}=\mu \Pi_{s} \Pi_{s}^{*}+\nu \Pi_{u} \Pi_{u}^{*}$, therefore the first term in the right hand side of (7.8) can be written as $\mu\left|\Pi_{s}^{*}(x-\xi)\right|^{2}+\nu\left|\Pi_{u}^{*}(x-\xi)\right|^{2}$. Thus (7.7) does hold if $0<\mu<1 / C_{1}$ and $0<\nu<1 / C_{1}$.

Next we prove

Lemma 15. If $\Phi_{\mu}^{\nu}(x)$ and $\mu, \nu$ are as in Lemma 14 then $W(x)>\Phi_{\mu}^{\nu}(x)$ in $\overline{U^{\prime}(\xi)} \backslash\{\xi\}$, where $U^{\prime}(\xi) \subset U(\xi)$ is a neighborhood of $\xi$.

Proof. Since $\xi$ is a significant point, we have $W(x)=d_{\bar{H}}(x, \xi)$ in $U(\xi)$. Due to the representation formula (2.11) $d_{\bar{H}}(x, \xi)=\lim _{k \rightarrow \infty} \int_{0}^{t^{k}} \bar{L}\left(\dot{\eta}^{k}, \eta^{k}\right) \mathrm{d} \tau$ for a sequence $t^{k}>0$ and absolutely continuous curves $\eta^{k}:\left[0, t^{k}\right] \rightarrow \bar{\Omega}$ satisfying the initial and terminal conditions $\eta^{k}(0)=\xi$, $\eta^{k}\left(t^{k}\right)=x$. We claim that there is a neighborhood $U^{\prime}(\xi) \subset U(\xi)$ such that $\forall x \in U^{\prime}(\xi)$ we have $\eta^{k}(\tau) \in U(\xi) \forall \tau \in\left[0, t^{k}\right]$ when $k$ is sufficiently large. Indeed, otherwise there are sequences of points $x^{k} \rightarrow \xi$ and curves $\eta^{k}(t), \eta^{k}(0)=\xi, \eta^{k}\left(t^{k}\right)=x^{k}$ that exit $U(\xi)$ at a time $t=\tau^{k}$ and $\lim _{k \rightarrow \infty} \int_{0}^{t^{k}} \bar{L}\left(\dot{\eta}^{k}, \eta^{k}\right) \mathrm{d} \tau=0$. Let us set $y^{k}:=\eta^{k}\left(\tau^{k}\right) \in \partial U^{\prime}(\xi)$, then $\lim _{k \rightarrow \infty} S_{\bar{H}}\left(y^{k}, \xi\right)=0$ and after extracting a subsequence $y^{k} \rightarrow y \in \partial U^{\prime}(\xi)$ we obtain $S_{\bar{H}}(y, \xi)=0$. Therefore $y \in \mathcal{A}_{\bar{H}}$. We can repeat this reasoning to find $y \in \mathcal{A}_{\bar{H}} \cap \partial U^{\prime \prime}(\xi)$ for every open subset $U^{\prime \prime}(\xi)$ of $U(\xi)$ containing point $\xi$. Thus $\xi$ cannot be isolated point of $\mathcal{A}_{\bar{H}}$, contradicting (2.17).

Now using (7.7) we get, for every $x \in U^{\prime}(\xi)$

$$
\begin{aligned}
\Phi_{\mu}^{\nu}(x)=\int_{0}^{t^{k}} \nabla \Phi_{\mu}^{\nu}\left(\eta^{k}\right) \cdot \dot{\eta}^{k} \mathrm{~d} \tau=\int_{0}^{t^{k}}\left(\nabla \Phi_{\mu}^{\nu}\left(\eta^{k}\right) \cdot \dot{\eta}^{k}-\bar{H}\left(\nabla \Phi_{\mu}^{\nu}\left(\eta^{k}\right), \eta^{k}\right)\right) \mathrm{d} \tau & \\
& +\int_{0}^{t^{k}} \bar{H}\left(\nabla \Phi_{\mu}^{\nu}\left(\eta^{k}\right), \eta^{k}\right) \mathrm{d} \tau \leq \int_{0}^{t^{k}} \bar{L}\left(\dot{\eta}^{k}, \eta^{k}\right) \mathrm{d} \tau
\end{aligned}
$$

when $k$ is sufficiently large. It follows that $\Phi_{\mu}^{\nu} \leq W$ in $U^{\prime}(\xi)$. On the other hand if $\Phi_{\mu}^{\nu}=W$ at a point $x_{0} \in U^{\prime}(\xi)$ then $x_{0}$ is a local minimum of $W-\Phi_{\mu}^{\nu}$ and $\bar{H}\left(\nabla \Phi_{\mu}^{\nu}\left(x_{0}\right), x_{0}\right) \geq 0$ since $W$ is a viscosity solution of $\bar{H}(\nabla W(x), x)=0$ in $\Omega$. Therefore $x_{0}=\xi$ by (7.7), i.e. $\Phi_{\mu}^{\nu}<W$ in $U^{\prime}(\xi) \backslash\{\xi\}$ and by choosing, if necessary, a smaller $U^{\prime}(\xi)$ we are done.

The following is the crucial step in establishing (7.3). We construct a test function $\Psi_{\varepsilon}(x)$ of the form $\Psi_{\varepsilon}(x)=\Phi_{\mu}^{\nu}(x)-\varepsilon \tilde{\theta}_{\varepsilon}(x, x / \varepsilon)$ which satisfies

$$
-\varepsilon a^{i j}(x, x / \varepsilon) \frac{\partial^{2} \Psi_{\varepsilon}}{\partial x_{i} \partial x_{j}}+H\left(\nabla \Psi_{\varepsilon}(x), x, x / \varepsilon\right) \leq \bar{H}\left(\nabla \Phi_{\mu}^{\nu}(x), x\right)+C \varepsilon \quad \text { in } U^{\prime}(\xi) .
$$


Assuming first that the solution $\vartheta(p, x, y)$ of (2.4), normalized by $\int_{Y} \vartheta(p, x, y) \mathrm{d} y=1$, is sufficiently smooth, we set $\tilde{\theta}_{\varepsilon}(x, y)=\theta\left(\nabla \Phi_{\mu}^{\nu}(x), x, y\right)$, where $\theta(p, x, y)=\log \vartheta(p, x, y)$. Then, since

$$
-a^{i j}(x, y) \frac{\partial^{2} \theta(p, x, y)}{\partial y_{i} \partial y_{j}}+H\left(p+\nabla_{y} \theta(p, x, y), x, y\right)=\bar{H}(p, x),
$$

one easily shows (7.9). Note that in this case $\tilde{\theta}_{\varepsilon}(x, y)$ is independent of $\varepsilon$. In the general case, thanks to $C^{1}$-regularity of the coefficients $a^{i j}(x, y)$ and $b^{j}(x, y)$, all the first and second order partial derivatives of $\vartheta(p, x, y)$ exist and continuous on $\mathbb{R}^{N} \times \bar{\Omega} \times \mathbb{R}^{N}$, except (possibly) $\partial^{2} \vartheta(p, x, y) / \partial x_{i} \partial x_{j}$. To obtain sufficient regularity of $\tilde{\theta}_{\varepsilon}(x, y)$ we set

$$
\tilde{\theta}_{\varepsilon}(x, y)=\int \varphi_{\varepsilon}\left(x-x^{\prime}\right) \theta\left(\nabla \Phi_{\mu}^{\nu}(x), x^{\prime}, y\right) \mathrm{d} x^{\prime},
$$

where $\varphi_{\varepsilon}(x)=\varepsilon^{-N} \varphi(x / \varepsilon)$, with $\varphi(x)$ being a smooth compactly supported nonnegative function and $\int \varphi(x) \mathrm{d} x=1$. Then we have

$$
a^{i j}(x, x / \varepsilon)\left(\frac{\partial^{2} \theta}{\partial y_{i} \partial y_{j}}\left(\nabla \Phi_{\mu}^{\nu}(x), x, x / \varepsilon\right)-\frac{\partial^{2}}{\partial x_{i} \partial x_{j}}\left(\theta_{\varepsilon}(x, x / \varepsilon)\right)\right) \leq \frac{C}{\varepsilon}
$$

and $\left|\nabla_{y} \theta\left(\nabla \Phi_{\mu}^{\nu}(x), x, x / \varepsilon\right)-\nabla\left(\theta_{\varepsilon}(x, x / \varepsilon)\right)\right| \leq C$. This eventually leads to (7.9).

It follows from (7.9) and (77.7) that

$$
-\varepsilon a^{i j}(x, x / \varepsilon) \frac{\partial^{2} \Psi_{\varepsilon}}{\partial x_{i} \partial x_{j}}+H\left(\nabla \Psi_{\varepsilon}(x), x, x / \varepsilon\right) \leq-\delta|x-\xi|^{2}+C \varepsilon \quad \text { in } U^{\prime}(\xi) .
$$

Consider now the function $W_{\varepsilon}-\Psi_{\varepsilon}$. By Lemma 15 we have $W_{\varepsilon}>\Psi_{\varepsilon}$ on $\partial U^{\prime}(\xi)$ for sufficiently small $\varepsilon$, therefore either $W_{\varepsilon} \geq \Psi_{\varepsilon}$ in $U^{\prime}(\xi)$ or $W_{\varepsilon}-\Psi_{\varepsilon}$ attains its negative minimum in $U^{\prime}(\xi)$ at a point $x_{\varepsilon}$. In the latter case we have $\nabla W_{\varepsilon}\left(x_{\varepsilon}\right)=\nabla \Psi_{\varepsilon}\left(x_{\varepsilon}\right)$ and $a^{i j}\left(x_{\varepsilon}, x_{\varepsilon} / \varepsilon\right) \frac{\partial^{2} W_{\varepsilon}}{\partial x_{i} \partial x_{j}}\left(x_{\varepsilon}\right) \geq$ $a^{i j}\left(x_{\varepsilon}, x_{\varepsilon} / \varepsilon\right) \frac{\partial^{2} \Psi_{\varepsilon}}{\partial x_{i} \partial x_{j}}\left(x_{\varepsilon}\right)$, this yields

$$
\begin{aligned}
\lambda_{\varepsilon}=-\varepsilon a^{i j}\left(x_{\varepsilon}, x_{\varepsilon} / \varepsilon\right) \frac{\partial^{2} W_{\varepsilon}}{\partial x_{i} \partial x_{j}}\left(x_{\varepsilon}\right)+H\left(\nabla W_{\varepsilon}\left(x_{\varepsilon}\right), x_{\varepsilon}, x_{\varepsilon} / \varepsilon\right) \\
\quad \leq-\varepsilon a^{i j}\left(x_{\varepsilon}, x_{\varepsilon} / \varepsilon\right) \frac{\partial^{2} \Psi_{\varepsilon}}{\partial x_{i} \partial x_{j}}\left(x_{\varepsilon}\right)+H\left(\nabla \Psi_{\varepsilon}\left(x_{\varepsilon}\right), x_{\varepsilon}, x_{\varepsilon} / \varepsilon\right) \leq-\delta\left|x_{\varepsilon}-\xi\right|^{2}+C \varepsilon .
\end{aligned}
$$

Thus either $W_{\varepsilon}>\Psi_{\varepsilon}$ in $U^{\prime}(\xi)$ or $W_{\varepsilon} \geq \Psi_{\varepsilon}+W_{\varepsilon}\left(x_{\varepsilon}\right)-\Psi_{\varepsilon}\left(x_{\varepsilon}\right)$ in $U^{\prime}(\xi)$ and $x_{\varepsilon}$ satisfies $\left|x_{\varepsilon}-\xi\right| \leq$ $C \sqrt{\varepsilon}$. Both cases lead to the bound $W_{\varepsilon}(x) \geq \Phi_{\mu}^{\nu}(x)+W_{\varepsilon}\left(\tilde{x}_{\varepsilon}\right)-\beta \varepsilon$, where $\tilde{x}_{\varepsilon}$ is either $\xi$ or $x_{\varepsilon}$ (recall that $u_{\varepsilon}$ is normalized by $u_{\varepsilon}(\xi)=1$, i.e. $\left.W_{\varepsilon}(\xi)=0\right)$. Then setting $z=(x-\xi) / \sqrt{\varepsilon}$ we get

$$
w_{\varepsilon}(z) \leq C w_{\varepsilon}\left(z_{\varepsilon}\right) e^{-\mu \phi_{s}(z)+\nu \phi_{u}(z)} \quad \text { in }(U(\xi)-\xi) / \sqrt{\varepsilon},
$$

where $z_{\varepsilon}=\left(\tilde{x}_{\varepsilon}-\xi\right) / \sqrt{\varepsilon}$ and hence $\left|z_{\varepsilon}\right| \leq C$. Observe that since $z_{\varepsilon}$ stay in a fixed compact as $\varepsilon \rightarrow 0$, then $w_{\varepsilon}\left(z_{\varepsilon}\right) \leq C$ and in the limit we therefore obtain

$$
w(z) \leq C e^{-\mu \phi_{s}(z)+\nu \phi_{u}(z)} \quad \text { in } \mathbb{R}^{N} .
$$


It remains to note that $\phi_{s}(z) \geq \gamma_{3}\left|\Pi_{s}^{*} z\right|^{2}$ and $\phi_{u}(z) \leq \gamma_{4}\left|\Pi_{u}^{*} z\right|^{2}$ for some $\gamma_{3}, \gamma_{4}>0$. Hence $w(z)$ does satisfy (7.3), and by Lemma 13 we have $\sigma=-2 \operatorname{tr}(\Gamma Q)=\sigma(\xi)$. Thus

$$
\limsup _{\varepsilon \rightarrow 0} \lambda_{\varepsilon} / \varepsilon=\sigma(\xi) \quad \text { for every significant point } \xi
$$

Inequalities (6.8) and (7.11) prove formula (2.18). Moreover they imply the uniqueness of the limiting additive eigenfunction $W(x)$, provided that the maximum in (2.18) is attained at exactly one $\xi=\bar{\xi}$. Indeed, we know that, up to extracting a subsequence, functions $W_{\varepsilon}$ converge uniformly (on compacts in $\Omega$ ) to an additive eigenfunction $W(x)$; here $W_{\varepsilon}=-\varepsilon \log u_{\varepsilon}$ and $u_{\varepsilon}$ are referred to the eigenfunctions normalized by (2.2). By (7.11) the unique significant point (associated to the chosen subsequence) is $\bar{\xi}$. Therefore $\bar{\xi}$ is the only minimal element in $\mathcal{A}_{\bar{H}}$ with respect to the order relation $\preceq$ defined in (17.1); hence it is the least element of $\mathcal{A}_{\bar{H}}$, i.e. $\bar{\xi} \preceq \xi$ for every $\xi \in \mathcal{A}_{\bar{H}}$. This means that $W(\xi)=W(\bar{\xi})+d_{\bar{H}}(\xi, \bar{\xi}) \forall \xi \in \mathcal{A}_{\bar{H}}$, and consequently $W(x)=d_{\bar{H}}(x, \bar{\xi})+W(\bar{\xi})$. Finally, by Corollary 11 we have $W(\bar{\xi})=0$, and Theorem 2 is now completely proved.

\section{Other scalings}

Theorem 2 can be generalized to the case of $\varepsilon^{\alpha}$-scaling, $\alpha>1$, of the fast variable in (1.1) (with $c(x, y)=0$ ). In this case the effective drift is still defined by formula (2.15) where $\theta^{*}$ is

now the $Y$-periodic solution of the equation $\frac{\partial^{2}}{\partial y_{i} \partial y_{j}}\left(a^{i j}(x, y) \theta^{*}(x, y)\right)=0$ normalized by $\int_{Y} \theta^{*} \mathrm{~d} y$. However, the discontinuous dependence of the effective drift on the parameter $\alpha \geq 1$ (at $\alpha=1$ ) might lead to a significant shift of the concentration set of the eigenfunction $u_{\varepsilon}$ from points $\xi$ where $\bar{b}(\xi)=0$, if $\alpha>1$ sufficiently close to 1 .

We outline main changes to be made in order to adapt the arguments of Sections 6 and 7 to the case $\alpha>1$. First of all let us introduce the approximate Hamiltonian $\bar{H}_{\varepsilon}(p, x)$ as the (additive) eigenvalue corresponding to a $Y$-periodic eigenfunction of

$$
-a^{i j}(x, y) \frac{\partial^{2} \theta_{\varepsilon}(p, x, y)}{\partial y_{i} \partial y_{j}}+H\left(p+\varepsilon^{\alpha-1} \nabla_{y} \theta_{\varepsilon}(p, x, y), x, y\right)=\bar{H}_{\varepsilon}(p, x),
$$

and the approximate $\operatorname{drift} \bar{b}_{\varepsilon}(x)$ by

$$
\bar{b}_{\varepsilon}^{j}(x)=-\frac{\partial \bar{H}_{\varepsilon}}{\partial p_{j}}(0, x)
$$

The eigenvalue $\bar{H}_{\varepsilon}$ is unique and $\theta_{\varepsilon}$ is unique up to an additive constant, moreover $\theta_{\varepsilon}$ can be found as the scaled logarithmic transformation $\theta_{\varepsilon}=-\frac{1}{\varepsilon^{2(\alpha-1)}} \log \vartheta_{\varepsilon}$ of a positive $Y$-periodic eigenfunction of the problem

$$
\varepsilon^{2(1-\alpha)} a^{i j}(x, y) \frac{\partial^{2} \vartheta_{\varepsilon}}{\partial y_{i} \partial y_{j}}+\varepsilon^{1-\alpha}\left(b^{j}(x, y)-2 a^{i j}(x, y) p_{i}\right) \frac{\partial \vartheta_{\varepsilon}}{\partial y_{j}}+H(p, x, y) \vartheta_{\varepsilon}=\bar{H}_{\varepsilon}(p, x) \vartheta_{\varepsilon} .
$$


Similarly to the case $\alpha=1$ the $\operatorname{drift} \bar{b}_{\varepsilon}(x)$ can be equivalently defined by

$$
\bar{b}_{\varepsilon}(x)=\int_{Y} b(x, y) \theta_{\varepsilon}^{*}(x, y) \mathrm{d} y,
$$

via the $Y$-periodic solution $\theta_{\varepsilon}^{*}$ of the equation

$$
\frac{\partial^{2}}{\partial y_{i} \partial y_{j}}\left(a^{i j}(x, y) \theta_{\varepsilon}^{*}\right)-\varepsilon^{\alpha-1} \frac{\partial}{\partial y_{j}}\left(b^{j}(x, y) \theta_{\varepsilon}^{*}\right)=0 .
$$

It is clear that $\bar{b}_{\varepsilon} \rightarrow \bar{b}$ in $C^{1}$ topology, therefore if $\bar{b}$ has, say $n$, zeros in $\Omega$, where the hyperbolicity condition (for the ODE $\dot{x}=-\bar{b}(x)$ ) is satisfied, then $\bar{b}_{\varepsilon}$ has exactly $n$ zeros at the distance at most $O\left(\varepsilon^{\alpha-1}\right)$ from the corresponding zeros of $\bar{b}$. Then, to show the lower bound for eigenvalues one follows the lines of Section 6 with a zero $\xi_{\varepsilon}$ of $\bar{b}_{\varepsilon}$ in place of the corresponding zero $\xi$ of $\bar{b}$, and $\theta_{\varepsilon}^{*}$ in place of $\theta^{*}$. Note that although $\xi_{\varepsilon} \rightarrow \xi$ as $\varepsilon \rightarrow 0$, the distance between this two points might be of order $\varepsilon^{\alpha-1}$, so that in the local scale $\sqrt{\varepsilon}$ this distance tends to infinity. Nevertheless, up to the shift from $\xi$ to $\xi_{\varepsilon}$ the local analysis is exactly the same as in Section 6 . Let us emphasize that for $\alpha \in(1,3 / 2)$ the statement of of Lemma 12 remains valid only if at least one of zeros of $\bar{b}$ is an interior point of $\Omega$.

The argument of Section 7 can also be adapted to the case $\alpha>1$. As in the proof of the lower bound one finds equation (7.2) for the limit of rescaled functions $w_{\varepsilon}(z)=u_{\varepsilon}\left(\xi_{\varepsilon}+\sqrt{\varepsilon} z\right)$, while the construction of the functions $\Phi_{\mu}^{\nu}$ and $\Psi_{\varepsilon}$ is to be modified. One can linearize the drift $\bar{b}_{\varepsilon}$ at $\xi_{\varepsilon}$ and construct the quadratic function $\Phi_{\mu}^{\nu}$ (which now depends on $\varepsilon$ ) following Section 7 with $B_{\varepsilon}^{i j}=\frac{\partial \bar{b}^{j}}{\partial x_{i}}\left(\xi_{\varepsilon}\right)$ in place of $B^{i j}$; also, in the construction of the function $\Psi_{\varepsilon}$ one makes use of the eigenfunction $\theta_{\varepsilon}$ (cf. (8.1) ) and sets $\Psi_{\varepsilon}(x)=\Phi_{\mu}^{\nu}(x)+\varepsilon^{2 \alpha-1} \theta_{\varepsilon}\left(\nabla \Phi_{\mu}^{\nu}(x), x, x / \varepsilon^{\alpha}\right)$. Details are left to the reader.

Finally note that the case $\alpha<1$ remains completely open.

\section{Example}

Here we consider an example of an operator of the form (2.14) for which conditions (2.17) are fulfilled. Let $x^{y}(t)$ be a solution of the ODE $\dot{x}^{y}=-\bar{b}\left(x^{y}\right), x^{y}(0)=y$. We assume that

- The vector field $\bar{b}(x)$ has exactly three zeros $\xi^{1}, \xi^{2}, \xi^{3}$ in $\bar{\Omega}$. All of them are interior points of $\Omega$.

- $\xi^{1}$ and $\xi^{3}$ are stable hyperbolic points, that is eigenvalues of $\left(-\frac{\partial \bar{b}^{j}}{\partial x_{i}}\left(\xi^{1}\right)\right)_{i, j=\overline{1, N}}$ and $\left(-\frac{\partial \bar{b}^{j}}{\partial x_{i}}\left(\xi^{3}\right)\right)_{i, j=1, N}$ have negative real parts; $\xi^{2}$ is a hyperbolic point and $\sigma\left(\xi^{2}\right)>$ $\max \left\{\sigma\left(\xi^{1}\right), \sigma\left(\xi^{3}\right)\right\}$.

- The ODE $\dot{x}=-\bar{b}(x)$ does not have a solution with $\lim _{t \rightarrow+\infty} x(t)=\lim _{t \rightarrow-\infty} x(t)=\xi^{2}$.

- For every $y \in \bar{\Omega} \backslash \bigcup_{j=1}^{3}\left\{\xi^{j}\right\}$, either $\lim _{t \rightarrow-\infty} x^{y}(t)=\xi^{2}$, or $\inf \left\{t<0 ; x^{y}(t) \in \bar{\Omega}\right\}>-\infty$. 
One can easily check that under these assumptions the Aubry set $\mathcal{A}_{\bar{H}}$ coincides with $\bigcup_{j=1}^{3}\left\{\xi^{j}\right\}$. Hence, by Theorem 2, $W(x)=d_{\bar{H}}\left(x, \xi^{2}\right)$ and $\lambda_{\varepsilon}=\varepsilon \sigma\left(\xi^{2}\right)+\bar{o}(\varepsilon)$.

It is interesting to trace in this example the possible structure of the set $\mathcal{Z}=\{x \in$ $\Omega ; W(x)=0\}$. It depends on whether there are trajectories of the equation $\dot{x}=-\bar{b}(x)$ going from $\xi^{1}$ or $\xi^{3}$ to $\xi^{2}$, or not.

Let $\mathcal{Z}^{1}$ be the set of all points $y \in \bar{\Omega}$ such that $\lim _{t \rightarrow+\infty} x^{y}(t)=\xi^{1}$ and $\lim _{t \rightarrow-\infty} x^{y}(t)=\xi^{2}$, and let $\mathcal{Z}^{3}$ the set of all points $y \in \bar{\Omega}$ such that $\lim _{t \rightarrow+\infty} x^{y}(t)=\xi^{3}$ and $\lim _{t \rightarrow-\infty} x^{y}(t)=\xi^{2}$. It follows from (2.11) that $\mathcal{Z}=\left\{\xi^{2}\right\} \cup \overline{\mathcal{Z}^{1}} \cup \overline{\mathcal{Z}^{3}}$.

\section{Appendices}

\section{A Uniqueness of additive eigenfunction}

The following simple result is a uniqueness criterion for problem (1.3)-(1.4).

Proposition 16. Let $\lambda=\lambda_{\bar{H}}$ so that (1.3)-(1.4) has a solution $W$. Then $W$ is unique (up to an additive constant) if and only if $S_{\bar{H}-\lambda}(x, y)=0$ for all $x, y \in \mathcal{A}_{\bar{H}-\lambda}$, where $S_{\bar{H}-\lambda}(x, y)=$ $d_{\bar{H}-\lambda}(x, y)+d_{\bar{H}-\lambda}(y, x)$.

Proof. If $S_{\bar{H}-\lambda}(x, y)=0$ then $W(x)-W(y)=d_{\bar{H}-\lambda}(x, y)$, since $\forall x, y$ we have $W(x)-W(y) \leq$ $d_{\bar{H}-\lambda}(x, y)$. In particular, if $S_{\bar{H}-\lambda}(x, y)=0 \forall x, y \in \mathcal{A}_{\bar{H}-\lambda}$ then pick $\xi \in \mathcal{A}_{\bar{H}-\lambda}$ to get $W(x)=d_{\bar{H}-\lambda}(x, \xi)+W(\xi)$ on $\mathcal{A}_{\bar{H}-\lambda}$. Thus $W(x)=d_{\bar{H}-\lambda}(x, \xi)+W(\xi)$ in $\Omega$ (according to the representation formula (2.13) $)$, i.e. $W$ is unique up to an additive constant.

If there are two points $\xi, \xi^{\prime} \in \mathcal{A}_{\bar{H}-\lambda}$ such that $S_{\bar{H}-\lambda}\left(\xi, \xi^{\prime}\right)>0$, then $W_{0}(x)=d_{\bar{H}-\lambda}(x, \xi)$ and $W_{1}(x)=d_{\bar{H}-\lambda}\left(x, \xi^{\prime}\right)-d_{\bar{H}-\lambda}\left(\xi, \xi^{\prime}\right)$ are two solutions of (1.3) $-(1.4)$ and $0=W_{0}(\xi)=W_{1}(\xi)$, while $W_{0}\left(\xi^{\prime}\right)-W_{1}\left(\xi^{\prime}\right)=S_{\bar{H}-\lambda}\left(\xi, \xi^{\prime}\right)>0$.

\section{B Aubry set for small perturbations of a gradient field}

We outline here the proof of the claim stated in Remark 3. Consider a vector field $b(x, y)$ which is a $C^{1}$-small perturbation of $\nabla P(x)$, i.e. $\|b(x, y)-\nabla P(x)\|_{C^{1}}=\delta$ and $\delta$ is sufficiently small. Let us show that the Aubry set $\mathcal{A}_{\bar{H}}$ of the Hamiltonian $\bar{H}(p, x)$ given by $(2.4)$ (with $c(x, y)=0$ ) is exactly the set of zeros of $\bar{b}(x)$ in $\Omega$, provided that $\delta$ is sufficiently small and $P \in C^{2}(\bar{\Omega})$ is as in Remark 3 .

Without loss of generality we can assume that $\bar{H}(p, x)=\sum p_{i}^{2}-\bar{b}^{i}(x) p_{i}$, since the Aubry set of this Hamiltonian coincides with that of the effective Hamiltonian given by (2.4). Let us first find the Aubry set $\mathcal{A}_{H^{0}}$ of the Hamiltonian $H^{0}(p, x)=\sum p_{i}^{2}-p_{i} \frac{\partial P(x)}{\partial x_{i}}$. We calculate the corresponding Lagrangian $L^{0}(v, x)=\frac{1}{4}|v+\nabla P(x)|^{2}$ and use criterion (2.12). Let $\xi \in \mathcal{A}_{H^{0}}$, then there exist a sequence of absolutely continuous curves $\eta^{n}:\left[0, t^{n}\right] \rightarrow \bar{\Omega}, \eta^{n}(0)=\eta^{n}\left(t^{n}\right)=\xi$, 
such that $t^{n} \rightarrow \infty$ and $\lim _{n \rightarrow \infty} \int_{0}^{t^{n}}\left|\dot{\eta}^{n}+\nabla P\left(\eta^{n}\right)\right|^{2} \mathrm{~d} \tau=0$. This yields

$$
0=\lim _{n \rightarrow \infty} \int_{0}^{t^{n}}\left(\left|\dot{\eta}^{n}\right|^{2}+2 \nabla P_{\delta}\left(\eta^{n}\right) \cdot \dot{\eta}^{n}+\left|\nabla P\left(\eta^{n}\right)\right|^{2}\right) \mathrm{d} \tau=\lim _{n \rightarrow \infty} \int_{0}^{t^{n}}\left(\left|\dot{\eta}^{n}\right|^{2}+\left|\nabla P\left(\eta^{n}\right)\right|^{2}\right) \mathrm{d} \tau
$$

Therefore, $\eta^{n}(t) \rightarrow \xi$ uniformly on every fixed interval $[0, T]$. It follows that $\xi$ belongs to the set $K=\{x \in \Omega ; \nabla P(x)=0\}$. Clearly, we also have $K \subset \mathcal{A}_{H^{0}}$. Now note that the effective drift $\bar{b}(x)$ given by (2.15), can be written as $\bar{b}(x)=\nabla P(x)+\tilde{b}_{\delta}(x)$ with $C^{1}$-small $\tilde{b}_{\delta}(x),\left\|\tilde{b}_{\delta}\right\|_{C^{1}}=\bar{O}(\delta)$ as $\delta \rightarrow 0$. Thanks to the assumption on critical points of $P(x)$, zeros of $\bar{b}(x)$ are isolated and are close to $K$ when $\delta$ is sufficiently small. Moreover, if $\omega$ is a small neighborhood of $\xi \in K$ then $\bar{b}(x)$ vanishes at exactly one point $\xi_{\delta} \in \omega$ and $\left|\xi-\xi_{\delta}\right|=O(\delta)$. Therefore, we can define a $C^{2}$ function $P_{\delta}$ such that $\left|\nabla P_{\delta}(x)\right|>0$ in $\bar{\Omega} \backslash K_{\delta}$, where $K_{\delta}$ is the set of zeros of $\bar{b}(x)$, and $\left|\bar{b}(x)-\nabla P_{\delta}(x)\right|=g_{\delta}(x)\left|\nabla P_{\delta}(x)\right|$ with $\max _{x \in \bar{\Omega}} g_{\delta}(x)=\bar{O}(\delta)$ as $\delta \rightarrow 0$. This yields the following bound (for small $\delta$ )

$$
|v+\bar{b}(x)|^{2} \geq \frac{1}{2}|v|^{2}+2 \nabla P_{\delta}(x) \cdot v+V_{\delta}(x), \quad \forall v \in \mathbb{R}^{N}, x \in \bar{\Omega},
$$

where $V_{\delta}>0$ in $\bar{\Omega} \backslash K_{\delta}$. Then, arguing as above we see that $\mathcal{A}_{\bar{H}}=K_{\delta}$. Moreover, every $\xi \in K_{\delta}$ is a hyperbolic fixed point of the ODE $\dot{x}=-\bar{b}(x)$, as $\delta$ is sufficiently small.

\section{Properties of solutions of Bernoulli matrix equation}

We provide here some results on Bernoulli equation (6.7), used in Sections 6] and 7. Recall that the matrix $Q$ in (6.7) is positive definite, $\Pi_{s}$ and $\Pi_{u}$ denote spectral projectors on the invariant subspaces of the matrix $B$ corresponding to eigenvalues with positive and negative real parts.

Proposition 17. The maximal positive semi-definite solution $\Gamma$ of (6.7) possesses the following properties: (i) $\Gamma=\Pi_{s} \Gamma \Pi_{s}^{*}, \quad$ (ii) $\Gamma \geq \gamma \Pi_{s} \Pi_{s}^{*}$ (in the sense of quadratic forms) for some $\gamma>0$, (iii) $2 \operatorname{tr}(Q \Gamma)=\operatorname{tr}\left(B \Pi_{s}\right)$, i.e. $2 \operatorname{tr}(Q \Gamma)$ is the sum of positive real parts of eigenvalues of $B$.

Proof. It follows from (6.7) that $X=\Pi_{u} \Gamma \Pi_{u}^{*}$ satisfies

$$
4 \Pi_{u} \Gamma Q \Gamma \Pi_{u}^{*}-\left(B \Pi_{u}\right) X-X\left(B \Pi_{u}\right)^{*}=0
$$

Consider the symmetric solution of (C.1) given by

$$
\tilde{X}=\int_{0}^{\infty} Y(t) \mathrm{d} t
$$

where $Y(t)=-4 e^{B \Pi_{u} t} \Pi_{u} \Gamma Q \Gamma \Pi_{u}^{*} e^{\left(B \Pi_{u}\right)^{*} t}$ (note that $\dot{Y}(t)=\left(B \Pi_{u}\right) Y(t)+Y(t)\left(B \Pi_{u}\right)^{*}$ and $Y(t) \rightarrow 0$ as $t \rightarrow+\infty$, therefore integrating we get $\left(B \Pi_{u}\right) \tilde{X}+\tilde{X}\left(B \Pi_{u}\right)^{*}=-Y(0)=4 \Pi_{u} \Gamma Q \Gamma \Pi_{u}^{*}$, i.e. $\tilde{X}$ does solve (C.1) ). We claim that $X=\tilde{X}$. Otherwise $Z:=X-\tilde{X}$ is a nonzero solution of equation $\left(B \Pi_{u}\right) Z+Z\left(B \Pi_{u}\right)^{*}=0$ and $Z=\Pi_{u} Z \Pi_{u}^{*}$. Then $Z(t)=Z$ is a stationary solution 
of the differential equation $\dot{Z}(t)=\left(B \Pi_{u}\right) Z(t)+Z(t)\left(B \Pi_{u}\right)^{*}$. The latter equation has the solution $\tilde{Z}(t)=e^{B \Pi_{u} t} \Pi_{u} Z \Pi_{u}^{*} e^{\left(B \Pi_{u}\right)^{*} t}$ which vanishes as $t \rightarrow+\infty$ and satisfies the initial condition $\tilde{Z}(0)=Z$. Thus $Z=0$, i.e. $X=\tilde{X}$. On the other hand it follows from (C.2) that $\tilde{X} \leq 0$ while $X \geq 0$, this yields $X=\tilde{X}=0$. Since $\Gamma$ is positive semi-definite we also have $\Pi_{u} \Gamma=\Gamma \Pi_{u}^{*}=0$ and the calculation $\Gamma=\left(\Pi_{u}+\Pi_{s}\right) \Gamma\left(\Pi_{u}+\Pi_{s}\right)^{*}=\Pi_{s} \Gamma \Pi_{s}^{*}$ shows (i). As a bi-product we also have established that $\Gamma$ is the maximal positive semi-definite solution of

$$
4 \Gamma Q \Gamma-\left(B \Pi_{s}\right) \Gamma-\Gamma\left(B \Pi_{s}\right)^{*}=0
$$

Indeed, assuming that $\tilde{\Gamma}$ is another positive semi-definite solution of $(\underline{C} .3)$ we get $\Pi_{u} \tilde{\Gamma} Q \tilde{\Gamma} \Pi_{u}^{*}=0$. This yields $\tilde{\Gamma} \Pi_{u}^{*}=0$ so that $\tilde{\Gamma}=\Pi_{s} \tilde{\Gamma} \Pi_{s}^{*}$, therefore $B \tilde{\Gamma}=B\left(\Pi_{s}\right)^{2} \tilde{\Gamma} \Pi_{s}^{*}=\left(B \Pi_{s}\right) \tilde{\Gamma}$ and $\tilde{\Gamma}$ thus solves (6.7).

To show (ii) and (iii) consider the maximal positive definite solution $\tilde{\Gamma}_{\delta}$ of

$$
4 \tilde{\Gamma}_{\delta} Q \tilde{\Gamma}_{\delta}-\left(B \Pi_{s}+\delta I\right) \tilde{\Gamma}_{\delta}-\tilde{\Gamma}_{\delta}\left(B \Pi_{s}+\delta I\right)^{*}=0
$$

for $\delta>0$. The existence of the unique positive definite solution follows from the fact that $\tilde{\Gamma}_{\delta}^{-1}$ is the unique solution of the Lyapunov matrix equation

$$
4 Q-\tilde{\Gamma}_{\delta}^{-1}\left(B \Pi_{s}+\delta I\right)-\left(B \Pi_{s}+\delta I\right)^{*} \tilde{\Gamma}_{\delta}^{-1}=0
$$

given by

$$
\tilde{\Gamma}_{\delta}^{-1}=4 \int_{-\infty}^{0} e^{\left(B \Pi_{s}+\delta I\right)^{*} t} Q e^{\left(B \Pi_{s}+\delta I\right) t} \mathrm{~d} t .
$$

It is known (see [28]) that $\tilde{\Gamma}_{\delta}$ converges to the (maximal positive semi-definite) solution $\Gamma$ of (C.3) as $\delta \rightarrow+0$. This allows to establish (iii) easily,

$$
2 \operatorname{tr}(Q \Gamma)=2 \lim _{\delta \rightarrow+0} 2 \operatorname{tr}\left(Q \tilde{\Gamma}_{\delta}\right)=\frac{1}{2} \lim _{\delta \rightarrow+0} \operatorname{tr}\left(\tilde{\Gamma}_{\delta}^{-1}\left(B \Pi_{s}+\delta I\right) \tilde{\Gamma}_{\delta}+\left(B \Pi_{s}+\delta I\right)^{*}\right)=\operatorname{tr}\left(B \Pi_{s}\right) .
$$

Finally, if we assume that (ii) is false, then there is $\eta \in \mathbb{R}^{N}$ such that $\Gamma \eta=0$ while $\Pi_{s}^{*} \eta \neq 0$. Thanks to (i) the equality $\Gamma \eta=0$ implies that $\Gamma \Pi_{s}^{*} \eta=0$. On the other hand, $\Gamma\left(\lim _{\delta \rightarrow+0} \tilde{\Gamma}_{\delta}^{-1} \Pi_{s} \Pi_{s}^{*} \eta\right)=\Pi_{s} \Pi_{s}^{*} \eta$, where the $\operatorname{limit} \lim _{\delta \rightarrow+0} \tilde{\Gamma}_{\delta}^{-1} \Pi_{s} \Pi_{s}^{*} \eta$ exists, for $e^{\left(B \Pi_{s}+\delta I\right)^{*} t} Q e^{\left(B \Pi_{s}+\delta I\right) t} \Pi_{s} \Pi_{s}^{*} \eta$ decays exponentially fast as $t \rightarrow-\infty$, uniformly in $\delta \geq 0$. According the Fredholm alternative $\Pi_{s} \Pi_{s}^{*} \eta$ and $\Pi_{s}^{*} \eta$ must be orthogonal, yielding $\left|\Pi_{s}^{*} \eta\right|=0$. We obtained a contradiction showing that (ii) does hold.

Acknowledgements. Part of this work was done when V. Rybalko was visiting the Narvik University College. He is grateful for a warm hospitality and support of his visit. 


\section{References}

[1] Armstrong, S.N., Souganidis, P.E. Stochastic homogenization of Hamilton-Jacobi and degenerate Bellman equations in unbounded envieronments. J. Math. Pure Appl., to appear, preprint arXiv:1103.2814v 4 .

[2] Armstrong, S.N., Souganidis, P.E. Stochastic homogenization of $L^{\infty}$ variational problems, preprint arXiv:1109.2853v3.

[3] Aronson, D.G. Non-negative solutions of linear parabolic equations. Ann. Scuola Norm. Sup. Pisa, 22(3) (1968), 607-694.

[4] Barles, G. Solutions de viscosité des équations de Hamilton-Jacobi. Springer-Verlag, Berlin, 1994.

[5] Barles, G., Perthame, B. Comparison principle for Dirichlet-type Hamilton-Jacobi equations and singular perturbations of degenerated elliptic equations. Appl. Math. Optim., 21(1) (1990), 21-44.

[6] Bethuel, F., Brezis, H. Hélein, F. Asymptotics for the minimizers of a Ginzburg-Landau functional. Calc. Var. and Partial Differential Equations 1 ( 1993), 123-148.

[7] Caffarelli, L.A., Souganidis, P.E., Wang, L. Homogenization of fully nonlinear, uniformly elliptic and parabolic partial differential equations in stationary ergodic media. Comm. Pure Appl. Math. 58(3) (2005), 319-361.

[8] Caffarelli, Luis A., Souganidis, P.E. Rates of convergence for the homogenization of fully nonlinear uniformly elliptic PDE in random media. Invent. Math. 180(2) (2010), 301-360.

[9] Camilli, F., Cesaroni, A. A note on singular perturbation problems via Aubry-Mather theory. Discrete Contin. Dyn. Syst. 17(4) (2007), 807-819.

[10] Camilli, F., Cesaroni, A., Siconolfi, A. Randomly perturbed dynamical systems and AubryMather theory. Int. J. Dyn. Syst. Differ. Equ. 2(3-4) (2009), 139-169.

[11] Capdeboscq, Y. Homogenization of a diffusion equation with drift. C. R. Acad. Sci. Paris Ser. I Math. 327(9) (1998), 807-812.

[12] Capuzzo-Dolcetta, I., Lions, P.-L. Hamilton-Jacobi equations with state constraints. Trans. Amer. Math. Soc. 318(2) (1990), 643-683.

[13] Donato, P., Piatnitski, A. Averaging of nonstationary parabolic operators with large lower order terms. Multi scale problems and asymptotic analysis, 153-165, GAKUTO Internat. Ser. Math. Sci. Appl., 24, Gakkotosho, Tokyo, 2006. 
[14] Davini, A., Siconolfi, A. Metric techniques for convex stationary ergodic Hamiltonians. Calc. Var. Partial Differential Equations 40(3-4) (2011), 391-421.

[15] Eizenberg, A., Kifer, Yu. The asymptotic behavior of the principal eigenvalue in a singular perturbation problem with invariant boundaries. Probab. Theory Related Fields, 76(4), (1987), 439-476.

[16] Evans, L.C. The perturbed test function method for viscosity solutions of nonlinear PDE. Proc. R. Soc. Edinburgh Sect. A 111 (3-4) (1989), 359-375.

[17] Evans, L.C. Periodic homogenisation of certain fully nonlinear partial differential equations. Proc. Roy. Soc. Edinburgh Sect. A 120(3-4) (1992), 245-265.

[18] Evans, L.C., Ishii, H. A PDE approach to some asymptotic problems concerning random differential equation with small noise intensities, Ann. L'Institut H. Poincare 2 (1985), 1-20.

[19] Freidlin, M.I., Wentzell, A.D. Random perturbations of dynamical systems. Fundamental Principles of Mathematical Sciences, 260. Springer-Verlag, New York, 1984.

[20] Gilbarg, D., Trudinger, N.S. Elliptic Partial Differential Equations of Second Order. BerlinHeidelberg-New York-Tokyo, Springer-Verlag, 1983.

[21] Ishii, H., Koike, S. Remarks on elliptic singular perturbation problems. Appl. Math. Optim. 23(1) (1991), 1-15.

[22] Ishii, H., Mitake, H. Representation formulas for solutions of Hamilton-Jacobi equations with convex Hamiltonians. Indiana Univ. Math. J. 56(5) (2007), 2159-2183.

[23] Kifer, Yu., On the principal eigenvalue in a singular perturbation problem with hyperbolic limit points and circles. J.Differential Equations, 37(1), (1980), 108-139.

[24] Kifer, Yu., Stochastic stability of the topological pressure. J. Analyse Math., 38, (1980), 255-286.

[25] Kosygina, E. Homogenization of stochastic Hamilton-Jacobi equations: brief review of methods and applications. Stochastic analysis and partial differential equations pp. 189204, Contemp. Math., 429, Amer. Math. Soc., Providence, 2007.

[26] Kosygina, E., Rezakhanlou, F., Varadhan, S.R.S. Stochastic homogenization of HamiltonJacobi-Bellman equations. Comm. Pure Appl. Math. 59(10) (2006), 1489-1521.

[27] Krylov, N.V.; Safonov, M.V. A property of the solutions of parabolic equations with measurable coefficients. Izv. Akad. Nauk SSSR Ser. Mat. 44(1) (1980), 161-175. 
[28] Lancaster, P., Rodman, L. Algebraic Riccati equations. Oxford Science Publications. The Clarendon Press, Oxford University Press, New York, 1995.

[29] Lions, P.-L., Souganidis, P.E. Homogenization of degenerate second-order PDE in periodic and almost periodic environments and applications. Ann. I.H. Poincaré, AN 22 (2005), $667-677$.

[30] Lions , P.-L., Souganidis, P.E. Homogenization of "viscous" Hamilton-Jacobi equations in stationary ergodic media. Comm. Partial Differential Equations 30(1-3) (2005), 335-375.

[31] Lions, P.-L., Papanicolaou, G., Varanhan, S.R.S., Homogenization of Hamilton-Jacobi equations, unpublished work, 1987.

[32] Majda, A.J., Souganidis, P.E. Large-scale front dynamics for turbulent reaction-diffusion equations with separated velocity scales. Nonlinearity, 7(1994), 1-30.

[33] Mitake, H. Asymptotic solutions of Hamilton-Jacobi equations with state constraints. Appl. Math. Optim. 58(3) (2008), 393-410.

[34] Perthame, B. Perturbed dynamical systems with an attracting singularity and weak viscosity limits in Hamilton-Jacobi equations. Trans. Amer. Math. Soc. 317(2) (1990), 723-748.

[35] Piatnitski, A. Asymptotic Behaviour of the Ground State of Singularly Perturbed Elliptic Equations. Commun. Math. Phys. 197 (1998), 527-551.

[36] Piatnitski , A.L. Averaging a singularly perturbed equation with rapidly oscillating coefficients in a layer. Math. USSR, Sb. 49 (1984), 19-40.

[37] Pyatnitskii, A.L., Shamaev, A.S. On the asymptotic behavior of the eigenvalues and eigenfunctions of a nonselfadjoint operator in $\mathbb{R}^{n}$. J. Math. Sci. 120(3) (2004), 1411-1423.

[38] Rauch, J. Five problems: an introduction to the qualitative theory of partial differential equations. Partial differential equations and related topics pp. 355-369. Lecture Notes in Math., Vol. 446, Springer, Berlin, 1975.

[39] Rezakhanlou, F., Tarver, J.E. Homogenization for stochastic Hamilton-Jacobi equations. Arch. Ration. Mech. Anal. 151(4) (2000), 277-309.

[40] Soner, H.M. Optimal control with state-space constraint. I. SIAM J. Control Optim. 24 (3) (1986), 552-561.

[41] Souganidis, P.E. Stochastic homogenization of Hamilton-Jacobi equations and some applications. Asymptot. Anal. 20(1) (1999), 1-11.

[42] Vishik, M.I., Lyusternik, L.A. Regular degeneracy and boundary layer for linear differential equations with a small parameter, Usp. Mat. Nauk, 12(5), (1957), 3-122. 